J. Fluid Mech. (2011), vol. 676, pp. 546-571. (c) Cambridge University Press 2011. The online version of this article is published within an Open Access environment subject to the conditions of the Creative Commons Attribution-NonCommercial-ShareAlike licence <http:// creativecommons.org/licenses/by-nc-sa/2.5/>. The written permission of Cambridge University Press must be obtained for commercial re-use.

doi:10.1017/jfm.2011.69

\title{
Low Rossby limiting dynamics for stably stratified flow with finite Froude number
}

\author{
BETH A. WINGATE ${ }^{1} \dagger$, PEDRO EMBID ${ }^{2}$, \\ MIRANDA HOLMES-CERFON ${ }^{3}$ AND MARK A. TAYLOR \\ ${ }^{1}$ MS D413, Los Alamos National Laboratory, Los Alamos, NM 87544, USA \\ ${ }^{2}$ Department of Mathematics and Statistics, the University of New Mexico, Albuquerque, \\ NM 87131, USA \\ ${ }^{3}$ New York University, Courant Institute of Mathematical Sciences, New York, NY 10012, USA \\ ${ }^{4}$ Sandia National Laboratories, Albuquerque, NM 87185, USA
}

(Received 27 May 2009; revised 26 January 2011; accepted 8 February 2011;

first published online 27 April 2011)

In this paper, we explore the strong rotation limit of the rotating and stratified Boussinesq equations with periodic boundary conditions when the stratification is order 1 ([Rossby number] $R o=\epsilon$, [Froude number] $F r=O(1)$, as $\epsilon \rightarrow 0$ ). Using the same framework of Embid \& Majda (Geophys. Astrophys. Fluid Dyn., vol. 87, 1998, p. 1), we show that the slow dynamics decouples from the fast. Furthermore, we derive equations for the slow dynamics and their conservation laws. The horizontal momentum equations reduce to the two-dimensional Navier-Stokes equations. The equation for the vertically averaged vertical velocity includes a term due to the vertical average of the buoyancy. The buoyancy equation, the only variable to retain its three-dimensionality, is advected by all three two-dimensional slow velocity components. The conservation laws for the slow dynamics include those for the two-dimensional Navier-Stokes equations and a new conserved quantity that describes dynamics between the vertical kinetic energy and the buoyancy. The leading order potential enstrophy is slow while the leading order total energy retains both fast and slow dynamics. We also perform forced numerical simulations of the rotating Boussinesq equations to demonstrate support for three aspects of the theory in the limit $R o \rightarrow 0$ : (i) we find the formation and persistence of large-scale columnar Taylor-Proudman flows in the presence of $O(1)$ Froude number; after a spin-up time, (ii) the ratio of the slow total energy to the total energy approaches a constant and that at the smallest Rossby numbers that constant approaches 1 and (iii) the ratio of the slow potential enstrophy to the total potential enstrophy also approaches a constant and that at the lowest Rossby numbers that constant is 1 . The results of the numerical simulations indicate that even in the presence of the low wavenumber white noise forcing the dynamics exhibit characteristics of the theory.

Key words: quasi-geostrophic flows, rotating flows, stratified flows

\section{Introduction}

For planetary scale rotating and stratified fluid dynamics, Charney (1948) estimated the orders of magnitude of different terms in the Euler equations by using typical 
values of large scale atmospheric motion. From these arguments, which included approximate hydrostatic and geostrophic balance, he derived reduced sets of equations called the quasi-geostrophic equations (QG) that are widely used in idealised studies of oceanic and atmospheric dynamics. In addition to finding equations that govern the large scales, Charney also points out that the QG equations 'filtered out' the inconsequential fast waves from the large scale motions.

In the work of Embid \& Majda (1996, 1998), Majda \& Embid (1998), they showed that the QG limit ([Rossby number] $R o \rightarrow 0$, [Froude number] $F r \rightarrow 0$ and $\mathrm{Fr} / \mathrm{Ro}=f / N$ finite) can also be derived from the rotating Boussinesq equations with periodic boundary conditions, (2.8)-(2.9). Their asymptotic analysis relies on the separation of fast and slow time scales and incorporates the fast waves that were filtered out in Charney's analysis. The resulting limiting equations are obtained by averaging over the fast time scale and accounts for three-waves interactions of fast and slow waves. Moreover, a rigorous justification of this approach was given by a direct application of Schochet's method of cancellation of oscillations for hyperbolic equations, Schochet (1994). In these papers, taking $R o \rightarrow 0$ corresponds to geostrophic balance and taking $\mathrm{Fr} \rightarrow 0$ corresponds to hydrostatic balance. When both these parameters go to zero, the equations for the slow dynamics decouple from the fast and are Charney's QG equations.

In addition to the quasi-geostrophic regime described above, we consider the dynamics of two other dynamical regimes: (i) the strong stratification limit where the physics is dominated by strong stratification but has only weak rotational effects and (ii) the strong rotation limit where the physics is dominated by fast rotation but is only weakly stratified.

The first limit, the strong stratification limit, is thought to be important in geophysical fluid dynamics, see the review by Riley \& Lelong (2000), because it describes flows that occur at length scales between the large, quasi-geostrophic scales and the small scales where energy is dissipated. Fluid dynamical theory for this physical regime has been explored by Riley \& Lelong (2000), Riley \& deBruynKops (2003), Babin, Mahalov \& Nicolaenko (1996), Babin et al. (1997), Babin, Mahalov \& Nicolaenko (1998, 2002), Embid \& Majda (1998). Parametrically this regime is described by $\mathrm{Fr} \rightarrow 0, \operatorname{Ro}=O(1), f / N \rightarrow 0$. Here it has also been found that the slow dynamics decouples from the fast and that it leads to new equations for the slow dynamics that are not the $\mathrm{QG}$ equations derived by Charney.

One way the slow dynamics of the QG limit differs from the slow dynamics of the strong stratification limit is in the role of the zero frequency dispersive waves. To explore this we examine the eigenfrequencies of the non-dimensional linearised rotating Boussinesq equations, (2.8) and (2.9) in the absence of dissipative effects,

$$
\omega(\boldsymbol{k})= \pm \frac{\left(F^{2} m^{2}+R o^{2}\left|\boldsymbol{k}_{H}\right|^{2}\right)^{1 / 2}}{\operatorname{RoFr}|\boldsymbol{k}|}, \quad \omega(\boldsymbol{k})=0 \quad \text { (double) }
$$

where $\left|\boldsymbol{k}_{H}\right|^{2}=k^{2}+l^{2}$ with $k$ and $l$ the horizontal wavenumbers, $m$ the vertical wavenumber and $|\boldsymbol{k}|^{2}=k^{2}+l^{2}+m^{2}$. There are two kinds of eigenfrequencies. The first kind are the slow vortical modes that have zero frequency for all $\boldsymbol{k}$, and contribute to the potential vorticity. The second kind are the dispersive waves that have non-zero frequency but make no contribution to potential vorticity. The latter are the familiar inertia-gravity waves that are filtered from Charney's QG equations. In the strong stratification limit (see Babin et al. 1997; Embid \& Majda 1998), where 
$F r \rightarrow 0, R o=O(1), f / N \rightarrow 0$, the fast eigenfrequencies are

$$
\omega_{F r}(\boldsymbol{k})= \pm \frac{\left|\boldsymbol{k}_{H}\right|}{|\boldsymbol{k}|}, \quad \omega(\boldsymbol{k})=0 \quad \text { (double). }
$$

Again there are two kinds of eigenfrequencies, the slow vortical modes and the fast gravity waves. However, here the fast waves contribute to the slow dynamics when $\boldsymbol{k}_{H}=0$. That is, one of the wave modes, corresponding to horizontal averages with zero potential vorticity, has a slow component that resonates with the PV bearing vortical modes. This manifests itself in the vertically sheared horizontal dynamics mode introduced by Embid \& Majda (1998) and investigated by Smith \& Waleffe (2002), Majda \& Grote (1997).

In this work, we look at the strong rotation (low Rossby) limit where geostrophic balance dominates but the flow is only weakly stratified. These dynamics are parametrically described by the limit $R o \rightarrow 0, F r=O(1), f / N \rightarrow \infty$.

This physical regime is relevant in regions of the deep ocean where stratification is weak but rotational effects are dominant. For example, Van Haren \& Millot (2005) observe values of $N=0 \pm 0.4 f(2.5<f / N<\infty)$ in the deep Mediterranean Sea and argue that the dynamics in those regions are driven by both weak stratification and the horizontal components rotation. In fact, they observe non-hydrostatic motions with vertical velocities of the same order of magnitude as the horizontal. Another region of the world where strong rotation and weak stratification have been observed is in the deep Arctic Ocean. Measurements in the Beaufort Gyre by Timmermans, Melling \& Rainville (2007), Timmermans et al. (2010) show $f / N \approx 2$ above $2600 \mathrm{~m}$ and $f / N \approx \infty$ between the depths of 2600 and $3600 \mathrm{~m}$. One of the reasons these investigators give for studying the deep Arctic is that in their 2002 pilot study they discovered the dynamics to be unexpectedly active in the deep ocean. Weak stratification in the deep ocean at high latitudes has been noted for the North Atlantic and North Pacific in Emery, Lee \& Magaard (1984) where they compute mean profiles of density and Brunt-Väisäla frequency; in the deep waters of the Arctic Ocean by Jones, Rudels \& Anderson (1995); and in the Southern Ocean by Heywood, Garabato \& Stevens (2002). Furthermore, warm core eddies with depths of $1000 \mathrm{~m}$ or more have been observed in the Arctic by Woodgate et al. (2001).

In the limit of strong rotation and weak stratification $(R o \rightarrow 0, F r=O(1)$, $f / N \rightarrow \infty)$ the fast eigenfrequencies are

$$
\omega_{R o}(\boldsymbol{k})= \pm \frac{|m|}{|\boldsymbol{k}|}, \quad \omega(\boldsymbol{k})=0 \quad \text { (double) }
$$

Again, there are two kinds of frequencies corresponding to fast inertial waves and slow PV modes. Also, in this limit the non-PV bearing modes make a contribution to the slow dynamics, but this time it occurs when $m=0$, which corresponds to vertically averaged dynamics, which we refer to as Taylor-Proudman dynamics.

By using the general framework developed in Embid \& Majda (1998), we show that in the low Rossby number limit the horizontal and vertical dynamics decouple. In the horizontal, the slow equations are the two-dimensional Navier-Stokes equations along with two conservation laws, the horizontal kinetic energy and the vertical vorticity. In the case where the flow is not stratified this is consistent with other work Chen et al. (2005). The vertically averaged vertical momentum equation is an advection-diffusion like equation that couples to the buoyancy through its vertical average. The slow equation for the buoyancy is the only quantity that remains fully three-dimensional and is advected by all three components of the slow velocity. The 
slow equations for the vertical momentum and the buoyancy are coupled and give rise to new conservation laws for the coupled $w-\rho$ dynamics. We also show that the slow modes evolve independent of the fast and that the total energy is composed of both slow and fast components, while the potential enstrophy is, to leading order in the expansion parameter, purely slow. The reduced equations and their conservation laws are supported by numerical simulations using low wavenumber forcing. These slow equations are not quasi-geostrophic because they are non-hydrostatic.

\section{Non-local form of the Boussinesq equations}

The Boussinesq equations for flow moving at a constant rotation about the $\widehat{z}$ axis for vertically stratified flow is

$$
\begin{gathered}
\frac{\mathrm{D}}{\mathrm{D} t} \boldsymbol{v}+f \hat{z} \times \boldsymbol{v}+\rho_{0}^{-1} \rho g \hat{z}+\rho_{0}^{-1} \nabla p=v \Delta \boldsymbol{v}, \\
\frac{\mathrm{D}}{\mathrm{D} t} \rho-b w=\kappa \Delta \rho, \\
\nabla \cdot \boldsymbol{v}=0,
\end{gathered}
$$

where is the material derivative, $v=(u, v, w)$ is the Eulerian velocity, $p$ is the pressure and the total density $\tilde{\rho}$ has been decomposed into $\tilde{\rho}=\rho_{o}-b z+\rho$, where $\rho_{0}$ is a constant background reference value of the density, $b$ is the density gradient in the vertical and $\rho$ is the density fluctuation. We assume $b>0$ for stable stratification. The parameter $f$ is twice the frame rotation rate, $g$ is the acceleration of gravity, $v$ is the kinematic viscosity and $\kappa$ is the diffusion coefficient.

In order to distinguish the physical mechanisms of fast rotation from weak stratification, we use the same velocity and length scales for all three components of velocity and in all three dimensions. Therefore we non-dimensionalise using the following characteristic scales; $L$ is the length scale for the three spatial coordinates $\boldsymbol{x}=(x, y, z), U$ is the velocity scale and $L / U$ is the advective time scale. The scale for the density fluctuation is $b U / N$, where $N=\left(\mathrm{gb} / \rho_{o}\right)^{1 / 2}$ is the Brunt-Väisälä frequency. Then we arrive at the following non-dimensional quantities,

$$
R o=\frac{U}{f L}, \quad E u=\frac{P}{\rho U^{2}}, \quad R e=\frac{U L}{\nu}, \quad \operatorname{Pr}=\frac{v}{\kappa}, \quad F r=\frac{U}{N L},
$$

where $R o$ is the Rossby number, $F r$ is the Froude number, $E u$ is the Euler number, $R e$ is the Reynolds number and $P r$ is the Prandtl number. Then the non-dimensional Boussinesq equations for rotating and stratified flows are

$$
\begin{gathered}
\frac{\mathrm{D}}{\mathrm{D} t} \boldsymbol{v}+\frac{1}{R o} \hat{z} \times \boldsymbol{v}+E u \nabla p+\frac{1}{F r} \rho \hat{z}=\frac{1}{R e} \Delta \boldsymbol{v}, \\
\frac{\mathrm{D}}{\mathrm{D} t} \rho-\frac{1}{F r} w=\frac{1}{\operatorname{RePr}} \Delta \rho \quad \text { with } \nabla \cdot \boldsymbol{v}=0 .
\end{gathered}
$$

It is clear that $v$ and $\rho$ are the evolution variables in (2.5) and (2.6) and that the role of the pressure gradient term in the momentum equation is to enforce the incompressibility condition. By eliminating the pressure term, it is possible to recast the Boussinesq equations exclusively in terms of the evolution variables and at the same time to incorporate the incompressibility constraint. This equivalent formulation is, however, in non-local form. To write these equations in their non-local form take 
the divergence of the momentum equation to find the equation for the pressure,

$$
E u \nabla p=\nabla \Delta^{-1}\left(\frac{1}{R o} \hat{z} \cdot \omega-\frac{1}{F r} \frac{\partial \rho}{\partial z}-\nabla \cdot(\boldsymbol{v} \cdot \nabla v)\right),
$$

where $\Delta^{-1}$ is the inverse Laplacian operator and $\omega=\nabla \times v=(\xi, \eta, \omega)$ is the local vorticity. Substitute the equation for the pressure into (2.5) and (2.6) to get the non-local form of the Boussinesq equations,

$$
\begin{gathered}
\frac{\mathrm{D}}{\mathrm{D} t} \boldsymbol{v}+\frac{1}{R o} \hat{\boldsymbol{z}} \times \boldsymbol{v}+\nabla \Delta^{-1}\left(\frac{1}{R o} \hat{\boldsymbol{z}} \cdot \boldsymbol{\omega}-\frac{1}{F r} \frac{\partial \rho}{\partial z}-\nabla \cdot(\boldsymbol{v} \cdot \nabla \boldsymbol{v})\right)+\frac{1}{F r} \rho \hat{z}=\frac{1}{R e} \Delta \boldsymbol{v}, \\
\frac{\mathrm{D}}{\mathrm{D} t} \rho-\frac{1}{F r} w=\frac{1}{R e P r} \Delta \rho .
\end{gathered}
$$

These equations automatically incorporate the incompressibility condition. Indeed, taking the divergence of $(2.8)$ results in $\partial / \partial t(\nabla \cdot v)=1 / \operatorname{Re} \Delta(\nabla \cdot v)$, so that if $\nabla \cdot v$ is zero initially, then it remains zero for all time.

A quantity of fundamental importance is the potential vorticity $\tilde{q}=\omega_{a} \cdot \nabla \tilde{\rho}$, where $\boldsymbol{\omega}_{a}=\boldsymbol{\omega}+f \hat{z}$. Clearly $\tilde{q}=q-f b$, where the evolution of $q=f(\partial \rho / \partial z)-b \omega+\boldsymbol{\omega} \cdot \nabla \rho$ is given by Ertel's theorem,

$$
\frac{\mathrm{D} q}{\mathrm{D} t}=v \Delta \omega \cdot \nabla \rho+\kappa \nabla(\Delta \rho) \cdot \boldsymbol{\omega}_{a} .
$$

If we scale $q$ with $f b F r$ and $\omega$ with $f R o$, then the non-dimensional form of $q$ is

$$
q=\frac{\partial \rho}{\partial z}-\frac{R o}{F r} \omega+R o(\omega \cdot \nabla \rho)
$$

and the non-dimensional form of Ertel's equation for $q$ is

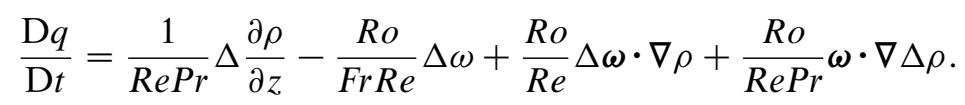

The equations for the global integrated total energy and potential enstrophy are

$$
\begin{aligned}
& \frac{1}{2} \frac{\mathrm{d}}{\mathrm{d} t} \int_{V}\left(|\boldsymbol{v}|^{2}+\rho^{2}\right) \mathrm{d} v=-\frac{1}{R e} \int_{V}|\nabla \boldsymbol{v}|^{2} \mathrm{~d} v-\frac{1}{\operatorname{RePr}} \int_{V}|\nabla \rho|^{2} \mathrm{~d} v, \\
& \frac{1}{2} \frac{\mathrm{d}}{\mathrm{d} t} \int_{V} q^{2} \mathrm{~d} v=\int_{V} q \frac{\partial q}{\partial t} \mathrm{~d} v=-\frac{1}{\operatorname{RePr}} \int_{V}\left|\nabla \frac{\partial \rho}{\partial z}\right|^{2} \mathrm{~d} v+O(\operatorname{Ro}) .
\end{aligned}
$$

The energy equation, (2.13) is independent of the Rossby and Froude numbers but the enstrophy equation (2.14) depends on the Rossby number, with a leading dissipative term depending on $|\nabla(\partial \rho / \partial z)|^{2}$ and the remaining contributions involving powers of the Rossby number.

\section{Limiting dynamics for the rapidly rotating Boussinesq equations}

Here we formulate the limiting dynamics for the rapidly rotating Boussinesq equations, i.e. in the limit of $R o \rightarrow 0$ and $F r=O(1)$. In doing so, we will follow the approach developed in great generality by Embid \& Majda (1998) and which builds upon earlier work of Klainerman \& Majda (1981), Majda (1984) and Schochet (1987, 1994). In fact, the present work complements the work of Embid and Majda which focused on the cases where $\mathrm{Fr} \rightarrow 0$ with either $R o / F r$ finite or $R o=O(1)$. The analysis starts with the recasting of the rotating Boussinesq equations in an abstract 
setting that reveals its key structure. This is followed with the asymptotic formulation of the slow dynamics equations in the limit of $R o \rightarrow 0$ and balanced initial data, i.e. without fast inertial waves. Finally, we adapt the theory developed by Embid and Majda to formulate limiting dynamics equations in the limit of $R o \rightarrow 0$ and with fast inertial waves.

\subsection{Abstract framework for the rotating Boussinesq equations}

If we introduce the vector $\boldsymbol{u}=(\boldsymbol{v}, \rho)$ and let $R o=\epsilon$, then the rotating Boussinesq equations, (2.8) and (2.9) become, in abstract operator form,

$$
\begin{gathered}
\frac{\partial \boldsymbol{u}}{\partial t}+\frac{1}{\epsilon} L_{F} \boldsymbol{u}+L_{S} \boldsymbol{u}+B(\boldsymbol{u}, \boldsymbol{u})=D \boldsymbol{u}, \\
\left.\boldsymbol{u}\right|_{t=0}=\boldsymbol{u}_{0}(\boldsymbol{x}),
\end{gathered}
$$

with the operators $L_{F}, L_{S}, B$ and $D$ given by

$$
\begin{gathered}
L_{F} \boldsymbol{u}=\left(\begin{array}{c}
\widehat{z} \times \boldsymbol{v}+\nabla \Delta^{-1} \omega \\
0
\end{array}\right) \\
L_{S} \boldsymbol{u}=(F r)^{-1}\left(\begin{array}{c}
\rho \widehat{z}-\nabla \Delta^{-1}\left(\frac{\partial \rho}{\partial z}\right) \\
-w
\end{array}\right) \\
B(\boldsymbol{u}, \boldsymbol{u})=\left(\begin{array}{c}
\boldsymbol{v} \cdot \nabla \boldsymbol{v}-\nabla \Delta^{-1}(\nabla \cdot(\boldsymbol{v} \cdot \nabla \boldsymbol{v})) \\
\boldsymbol{v} \cdot \nabla \rho \\
(\operatorname{Re})^{-1} \Delta \boldsymbol{v} \\
(\operatorname{Re})^{-1}(\operatorname{Pr})^{-1} \Delta \rho
\end{array}\right) .
\end{gathered}
$$

In the equations above the linear operator $L=\epsilon^{-1} L_{F}+L_{S}$ splits into a fast piece $L_{F}$ associated with the Rossby number $R o=\epsilon$ and a slow piece $L_{S}$ associated with the Froude number. It is clear that (3.1) becomes singular in the limit of $\epsilon \rightarrow 0$, and the fast operator $L_{F}$ will have a dominant role. The remaining terms in (3.1) are given by the bilinear advective operator $B(\boldsymbol{u}, \boldsymbol{u})$ and the diffusion operator $D \boldsymbol{u}$.

As we mentioned before, if the initial data $\boldsymbol{u}_{0}$ in (3.1) are divergence free, then the solution remains divergence free for all time. But in fact more is true, each individual operator, $L_{F}, L_{S}, B$ and $D$, takes solenoidal fields into solenoidal fields. Therefore, a natural setting for (3.1) is the Hilbert space $X$ of vector fields $\boldsymbol{u}=(\boldsymbol{v}, \rho)$ in $L^{2}$ that are divergence free, $\nabla \cdot v=0$, and equipped with the $L^{2}-$ norm, which is physically equivalent to the total energy, $\|\boldsymbol{u}\|^{2}=\int|\boldsymbol{v}|^{2}+\rho^{2} \mathrm{~d} v$. In addition, we assume $2 \pi$-periodicity in all the space variables. This choice of boundary conditions considerably simplifies the study of (3.1), particularly the analysis of the operator $L_{F}$, and the resulting slow limiting dynamics equations, (3.13), and the fast wave averaging equations, (3.32). The reason for this simplification is the fact that the associated eigenfunctions are given explicitly in terms of Fourier modes. In addition, the choice of periodic boundary conditions is consistent with the numerical simulations presented in $\S 4$. Changing the domain and the boundary conditions can make the mathematical analysis quite difficult; for example, for arbitrary bounded domains it may be impossible to characterise the eigenfunctions of $L_{F}$. The choice of an infinite domain may change drastically the structure of the null space (slow waves) and the range (fast waves) of the operator $L_{F}$. 
One key observation is the fact that the operator $L_{F}$ (and $L_{S}$ ) is skew-Hermitian in $X$ : for $\boldsymbol{u}_{1}$ and $\boldsymbol{u}_{2}$ in $X$,

$$
\int_{V} \boldsymbol{u}_{2}^{*} L_{F} \boldsymbol{u}_{1} \mathrm{~d} v=-\int_{V}\left(L_{F} \boldsymbol{u}_{2}\right)^{*} \boldsymbol{u}_{1} \mathrm{~d} v
$$

where $\boldsymbol{u}^{*}$ denotes the conjugate transpose of $\boldsymbol{u}$. Several important properties follow from this fact. First, (3.1) satisfies (in the absence of diffusion) the conservation of energy, (2.13). This property is shared with other important systems in mathematical physics, such as the Euler and the Maxwell equations. Second, according to the spectral theorem, skew-Hermitian operators have purely imaginary eigenvalues and an orthonormal basis of eigenfunctions, see Lax (2002). Physically this means that the basic normal mode solutions of equations represent wave motions. Finally, the null space of $L_{F}, N\left(L_{F}\right)$, is orthogonal to the range of $L_{F}, R\left(L_{F}\right)$. This can be thought of a consequence of the spectral theorem because $N\left(L_{F}\right)$ is spanned by the eigenfunctions with zero eigenvalue (slow modes) whereas $R\left(L_{F}\right)$ is spanned by the remaining eigenfunctions with non-zero eigenvalues (fast modes). This last property will be exploited later in the derivation of the slow dynamics equations.

Next we apply the previous observations to the linear equation,

$$
\frac{\partial \boldsymbol{u}}{\partial t}+\frac{1}{\epsilon} L_{F} \boldsymbol{u}=0
$$

and seek normal mode solutions in the form of harmonic plane waves

$$
\boldsymbol{u}(\boldsymbol{x}, t)=\boldsymbol{r} \exp \left[\mathrm{i} k \cdot x-\mathrm{i} \epsilon^{-1} \omega(\boldsymbol{k}) t\right]
$$

where $\boldsymbol{k}=(k, l, m)$ is the wavenumber, $\omega(\boldsymbol{k})$ is the frequency and the purely imaginary number $\lambda=\mathrm{i} \omega(\boldsymbol{k})$ is the eigenvalue of $L_{F}$ associated with the eigenfunction $\boldsymbol{u}=\boldsymbol{r} \exp [\mathrm{i} \boldsymbol{k} \cdot \boldsymbol{x}]$. The four eigenfrequencies $\omega(\boldsymbol{k})$ are given by the dispersion relations,

$$
\omega(\boldsymbol{k})= \pm m /|\boldsymbol{k}|, \quad \omega(\boldsymbol{k})=0 \quad(\text { double })
$$

Therefore, the equations admit slow modes moving on time scales $O(1)$ when $\omega(\boldsymbol{k})=0$ and fast waves moving on time scales $O(1 / \epsilon)$ when $\omega(\boldsymbol{k}) \neq 0$. The fast waves in this limit are gyroscopic or inertial waves. They are waves that owe their existence to the presence of the Coriolis force and were originally described by Kelvin (1880). Descriptions of these waves can be found in LeBlond \& Mysak (1978) and Greenspan (1990). Of course, if $m=0$ in (3.9) then we only have slow gyroscopic waves. Explicit formulas for the eigenvectors $\boldsymbol{r}$ associated with the fast and slow normal modes are given in the Appendix.

\subsection{Slow limiting dynamics as $R o \rightarrow 0$}

Here we consider the limiting dynamics equations as $R o \rightarrow 0$ under the assumption that the solution $\boldsymbol{u}^{\epsilon}(\boldsymbol{x}, t)$ of (3.1) evolves only on the slow (advective) time scale. The formal derivation in the context of the abstract operator equation, (3.1), is straightforward. We start by assuming that $\boldsymbol{u}^{\epsilon}(\boldsymbol{x}, t)$ has the asymptotic expansion,

$$
\boldsymbol{u}^{\epsilon}(\boldsymbol{x}, t)=\boldsymbol{u}^{0}(\boldsymbol{x}, t)+\epsilon \boldsymbol{u}^{1}(\boldsymbol{x}, t)+O\left(\epsilon^{2}\right),
$$

as $\epsilon \rightarrow 0$. Plugging $\boldsymbol{u}^{\epsilon}$ into (3.1) and collecting the contribution of order $O\left(\epsilon^{-1}\right)$ yields

$$
L_{F} \boldsymbol{u}^{0} \equiv 0
$$

that is, $\boldsymbol{u}^{0}$ is in $N\left(L_{F}\right)$ for all time, or equivalently, $\boldsymbol{u}^{0}(\boldsymbol{x}, t)$ is represented exclusively in terms of slow modes. In particular, the initial data $\boldsymbol{u}_{0}(\boldsymbol{x})=\boldsymbol{u}^{0}(\boldsymbol{x}, 0)$ are in $N\left(L_{F}\right)$ 
to leading order in $\epsilon$. The next contribution of order $O\left(\epsilon^{0}\right)$ yields

$$
\frac{\partial \boldsymbol{u}^{0}}{\partial t}+L_{F} \boldsymbol{u}^{1}+L_{S} \boldsymbol{u}^{0}+B\left(\boldsymbol{u}^{0}, \boldsymbol{u}^{0}\right)-D \boldsymbol{u}^{0}=0 .
$$

The slow limiting dynamics equation is now obtained by projecting (3.12) onto $N\left(L_{F}\right)$ as follows. First apply the orthogonal projection $P$ of $X$ onto $N\left(L_{F}\right)$ to both sides of (3.12). Since $\boldsymbol{u}^{0}$ is in $N\left(L_{F}\right)$ for all time so is $\partial \boldsymbol{u}^{0} / \partial t$, hence $P\left(\partial \boldsymbol{u}^{0} / \partial t\right)=\partial \boldsymbol{u}^{0} / \partial t$. In addition, since $L_{F} \boldsymbol{u}^{1}$ is in $R\left(L_{F}\right)$ and $N\left(L_{F}\right)$ is orthogonal to $R\left(L_{F}\right)$, then $P\left(L_{F} \boldsymbol{u}^{1}\right)=0$ and any contribution from $\boldsymbol{u}^{1}$ is eliminated under the projection. Finally, we eliminate the superscript in $\boldsymbol{u}^{0}$ and obtain the slow limiting dynamics equations,

$$
\frac{\partial \boldsymbol{u}}{\partial t}+P\left(L_{S} \boldsymbol{u}+B(\boldsymbol{u}, \boldsymbol{u})-D \boldsymbol{u}\right)=0,\left.\quad \boldsymbol{u}\right|_{t=0}=\boldsymbol{u}_{0}(\boldsymbol{x}) \in N\left(L_{F}\right) .
$$

Next we notice that, to leading order in $\epsilon$, it is enough for the initial data $\boldsymbol{u}_{0}(\boldsymbol{x})$ to be in $N\left(L_{F}\right)$ to automatically guarantee that the solution $\boldsymbol{u}(\boldsymbol{x}, t)$ of (3.13) remains in $N\left(L_{F}\right)$ for all time. Indeed, if we integrate in time (3.13) and use the fact that $\boldsymbol{u}_{0} \in N\left(L_{F}\right)$, we conclude that $\boldsymbol{u}(t) \in N\left(L_{F}\right)$ for all time. Moreover, we will show shortly that in the context of the rotating Boussinesq equations the null space $N\left(L_{F}\right)$ consists precisely of the Taylor-Proudman columnar flows, Taylor (1921). Therefore, we can say that to leading order in $\epsilon$, if the initial data are Taylor-Proudman flows (i.e. free of fast inertial waves), then the solution $\boldsymbol{u}$ remains a Taylor-Proudman flow for all time and its evolution is described by the slow dynamics equations. When the initial data are Taylor-Proudman flows to leading order in $\epsilon$, we say that the flow is in approximate Taylor-Proudman balance. Finally we remark that all these formal considerations can be established with full mathematical rigour through a direct application of the general theory of singular limits of hyperbolic systems first developed by Klainerman \& Majda (1981), Majda (1984), with later additions by Schochet (1987).

To obtain the concrete formulation of the slow dynamics for the Boussinesq equations, (3.1)-(3.5), we need to determine explicitly the null space $N\left(L_{F}\right)$ and its orthogonal projection $P$. For this purpose, it is convenient to split vectors and operators into their horizontal and vertical components. Thus, the velocity $\boldsymbol{v}=\left(\boldsymbol{v}_{H}, w\right)$ with $\boldsymbol{v}_{H}=(u, v)$, the gradient $\nabla=\left(\nabla_{H}, \partial / \partial z\right)$ with $\nabla_{H}=((\partial / \partial x),(\partial / \partial y))$, and the Laplacian $\Delta=\Delta_{H}+\left(\partial^{2} / \partial z^{2}\right)$ with $\Delta_{H}=\left(\partial^{2} / \partial x^{2}\right)+\left(\partial^{2} / \partial y^{2}\right)$.

The null space $N\left(L_{F}\right)$ of the fast operator $L_{F}$ in (3.2) is characterised by

$$
\begin{aligned}
-v+\frac{\partial}{\partial x} \Delta^{-1} \omega & =0, \\
u+\frac{\partial}{\partial y} \Delta^{-1} \omega & =0, \\
\frac{\partial}{\partial z} \Delta^{-1} \omega & =0,
\end{aligned}
$$

where $\omega=(\partial v / \partial z)-(\partial u / \partial y)$ is the vertical component of the vorticity and $\boldsymbol{v}$ is incompressible, $\boldsymbol{\nabla} \cdot \boldsymbol{v}=0$. From (3.16), it follows that $\Delta^{-1} \omega=\psi$ is $z$ independent of $z$. Introducing $\psi$ back into (3.14)-(3.15), shows that $\psi$ is the streamfunction for $\boldsymbol{v}_{H}, \boldsymbol{v}_{H}=(-(\partial \psi / \partial y), \partial \psi / \partial x)$, and that $\boldsymbol{v}_{H}$ is incompressible, $\nabla_{H} \cdot \boldsymbol{v}_{H}=(\partial / \partial x)(-(\partial \psi / \partial y))+(\partial / \partial y)(\partial \psi / \partial x)=0$. Since $\boldsymbol{v}$ is incompressible by assumption, then it follows that $(\partial w / \partial z)=0$, i.e. $w$ is also $z$-independent. This shows that $N\left(L_{F}\right)$ consists of Taylor-Proudman column flows, i.e. states $\boldsymbol{u}=(\boldsymbol{v}, \rho)$ with $\boldsymbol{v}$ $z$-independent and $\boldsymbol{v}_{H}$ incompressible. That no restrictions are imposed upon $\rho$ is 
not surprising since the fast operator $L_{F}$ only includes those contributions associated with the Rossby number.

The orthogonal projection $P$ onto the null space $N\left(L_{F}\right)$ is given by

$$
P \boldsymbol{u}=\left(\begin{array}{c}
\left\langle\boldsymbol{v}_{H}\right\rangle_{z}-\nabla_{H} \Delta_{H}^{-1}\left(\nabla_{H} \cdot\left\langle\boldsymbol{v}_{H}\right\rangle_{z}\right) \\
\langle w\rangle_{z} \\
\rho
\end{array}\right),
$$

where $\langle f\rangle_{z}=(1 / 2 \pi) \int_{0}^{2 \pi} f(x, y, z) d z$ denotes the average in the vertical direction. Therefore the concrete form of (3.13), the slow limiting dynamics equations for the rotating Boussinesq equations is

$$
\begin{array}{r}
\frac{\partial \boldsymbol{v}_{H}}{\partial t}+\boldsymbol{v}_{H} \cdot \nabla_{H} \boldsymbol{v}_{H}+\nabla_{H} p=\frac{1}{R e} \Delta_{H} \boldsymbol{v}_{H}, \\
\nabla_{H} \cdot \boldsymbol{v}_{H}=0, \\
\frac{\partial w}{\partial t}+\boldsymbol{v}_{H} \cdot \nabla_{H} w=\frac{1}{\operatorname{Re} \Delta_{H} w-\frac{1}{F r}\langle\rho\rangle_{z},} \\
\frac{\partial \rho}{\partial t}+\boldsymbol{v} \cdot \nabla \rho-\frac{1}{F r} w=\frac{1}{\operatorname{RePr}} \Delta \rho,
\end{array}
$$

where $\boldsymbol{v}=\boldsymbol{v}(x, y, t), \rho=\rho(x, y, z, t)$ and $\nabla_{H} p=\nabla_{H} \Delta_{H}^{-1}\left[\nabla_{H} \cdot\left(\boldsymbol{v}_{H} \cdot \nabla_{H} \boldsymbol{v}_{H}\right)\right]$. For brevity of notation we omit distinguishing between projected and unprojected variables and instead state that all the variables in (3.18)-(3.21) are the result of applying the projection operator, (3.17).

In the slow dynamics, the horizontal component of the velocity $\boldsymbol{v}_{H}$ is governed by the 2D Navier-Stokes equation. Moreover, $\boldsymbol{v}_{H}$ evolves independent of the vertical velocity $w$ and the density $\rho$ but it influences the dynamics of these variables through the advection terms in (3.20) and (3.21). The dynamics of the vertical velocity $w$ and the density $\rho$ are strongly coupled. Interestingly, the vertical velocity $w$ evolves according to a $2 \mathrm{D}$ forced advection-diffusion equation, (3.20), with buoyancy force given by $\langle\rho\rangle_{z}$, the density average in the vertical direction. On the other hand, the evolution of the density $\rho$ is given by the 3D forced advection-diffusion equation, (3.21), and remains the same as (2.6) in the Boussinesq approximation.

A consequence of this decoupling of the horizontal velocity from the vertical velocity and the density in (3.18)-(3.21) is the appearance of additional globally integrated conservation laws which are not present in the original Boussinesq equations. First, the 2D Navier-Stokes equation for $\boldsymbol{v}_{H}$ yields the conservation of horizontal kinetic energy and enstrophy,

$$
\begin{aligned}
\frac{1}{2} \frac{\mathrm{d}}{\mathrm{d} t} \int_{A}\left|\boldsymbol{v}_{H}\right|^{2} \mathrm{~d} a & =-\frac{1}{R e} \int_{A}\left|\nabla_{H} \boldsymbol{v}_{H}\right|^{2} \mathrm{~d} a, \\
\frac{1}{2} \frac{\mathrm{d}}{\mathrm{d} t} \int_{A} \omega^{2} \mathrm{~d} a & =-\frac{1}{R e} \int_{A}\left|\nabla_{H} \omega\right|^{2} \mathrm{~d} a,
\end{aligned}
$$

where integration is over the horizontal period square $A=[0,2 \pi]^{2}$. Next, taking the vertical average of the equation for $\rho$, (3.21), results in an evolution equation for $\langle\rho\rangle_{z}$,

$$
\frac{\partial}{\partial t}\langle\rho\rangle_{z}+\boldsymbol{v}_{H} \cdot \nabla_{H}\langle\rho\rangle_{z}-\frac{1}{F r} w=\frac{1}{\operatorname{RePr}} \Delta_{H}\langle\rho\rangle_{z} .
$$


Combining (3.20) and (3.24) results in horizontal conservation laws for the vertical kinetic energy and the average vertical potential energy,

$$
\begin{aligned}
\frac{1}{2} \frac{\mathrm{d}}{\mathrm{d} t} \int_{A} w^{2} \mathrm{~d} a & =-\frac{1}{F r} \int_{A} w\langle\rho\rangle_{z} \mathrm{~d} a-\frac{1}{\operatorname{Re}} \int_{A}\left|\nabla_{H} w\right|^{2} \mathrm{~d} a, \\
\frac{1}{2} \frac{\mathrm{d}}{\mathrm{d} t} \int_{A}\langle\rho\rangle_{z}^{2} \mathrm{~d} a & =\frac{1}{F r} \int_{A} w\langle\rho\rangle_{z} \mathrm{~d} a-\frac{1}{\operatorname{RePr}} \int_{A}\left|\nabla_{H}\langle\rho\rangle_{z}\right|^{2} \mathrm{~d} a,
\end{aligned}
$$

and adding these two equations results in the conservation of vertical energy,

$$
\frac{1}{2} \frac{\mathrm{d}}{\mathrm{d} t} \int_{A}\left(w^{2}+\langle\rho\rangle_{z}^{2}\right) \mathrm{d} a=-\frac{1}{\operatorname{Re}} \int_{A}\left|\nabla_{H} w\right|^{2} \mathrm{~d} a-\frac{1}{\operatorname{RePr}} \int_{A}\left|\nabla_{H}\langle\rho\rangle_{z}\right|^{2} \mathrm{~d} a .
$$

Additionally, if we define the density fluctuation $\tilde{\rho}=\rho-\langle\rho\rangle_{z}$, then from the density equations, (3.21)-(3.24), it follows the conservation of potential energy,

$$
\frac{1}{2} \frac{\mathrm{d}}{\mathrm{d} t} \int_{V} \tilde{\rho}^{2} \mathrm{~d} v=-\frac{1}{\operatorname{RePr}} \int_{V}|\nabla \tilde{\rho}|^{2} \mathrm{~d} v,
$$

where the volume integral is over the period cube $V=[0,2 \pi]^{3}$. Of course, (2.13) for the conservation of total energy still holds for the slow dynamics equations, (3.18)-(3.21). Finally, define the potential vorticity $q$ for the slow dynamics equations as the leading term of the expansion of $q$ given by (2.11) in powers of $R o=\epsilon, q=\partial \rho / \partial z=\partial \tilde{\rho} / \partial z$. Then the equation for the conservation of potential enstrophy is

$$
\frac{1}{2} \frac{\mathrm{d}}{\mathrm{d} t} \int_{V} q^{2} \mathrm{~d} v=-\frac{1}{\operatorname{RePr}} \int_{V}|\nabla q|^{2} \mathrm{~d} v .
$$

The conservation laws above were obtained directly from the slow limiting dynamics equations. In the different limiting regimes of strong stratification and Burger number $B u=(R o / F r)^{2}=0(1)$, or strong stratification and weak rotation, Babin et al. (1997, 1998) showed that conserved quantities for the limiting equations, like the horizontally averaged buoyancy correspond to adiabatic invariants for the full Boussinesq equations.

Finally, we remark that although the abstract derivation of the slow limiting dynamics equations, (3.13), is completely general, the concrete form of the equations depend on the explicit calculation of $N\left(L_{F}\right), R\left(L_{F}\right)$ and the projection operator $P$ of $X$ onto $N\left(L_{F}\right)$. This calculation is very much dependent on the choice of the domain and the boundary conditions. For example, if we assume the horizontal variables infinite in extent and periodicity in the vertical variable, i.e. $V=\mathbb{R}^{2} \times[0,2 \pi]$, then $N\left(L_{F}\right)$ is still given by Taylor-Proudman columns, (3.14)-(3.16), the projection operator by (3.17) and the slow limiting dynamics equations by (3.18)-(3.21). On the other hand, if we also assume infinite extent in the vertical variable, i.e. $V=\mathbb{R}^{3}$, then $N\left(L_{F}\right)$ only admits flows with zero velocity (this is reasonable because the only velocity field $v$ that is $z$-independent and has finite kinetic energy is $\boldsymbol{v}=\mathbf{0}$ ) and in this case the slow limiting dynamics becomes trivial. However, it is arguable whether a fluid of infinite depth constitutes a reasonable assumption in the present context.

\subsection{Fast limiting dynamics as $R o \rightarrow 0$}

The derivation of the slow limiting dynamics equations presented in the previous section was based on the assumption that the solution evolved only on the slow advective time scale. This assumption is warranted if, to leading order in $\epsilon$, the initial data do not include fast inertial waves components. If, on the other hand, the initial data contain inertial waves, then the limiting dynamics equations as $R o \rightarrow 0$ must 
be modified to take into account the fast inertial waves. The derivation of the fast limiting dynamics for small Rossby number and finite Froude number is readily obtained by invoking the very general approach developed in the fundamental work of Embid \& Majda (1998) on the limiting dynamics of the Boussinesq equations with small Froude number and either finite or small Rossby number. In fact, we only have to switch the roles of the fast and slow operators $L_{F}$ and $L_{S}$ and apply their theory in straightforward fashion. For this reason we are content with a summary of the main points of their theory and their most relevant conclusions for the present work.

Following Embid \& Majda (1998) we assume that the solution $\boldsymbol{u}^{\epsilon}(\boldsymbol{x}, t)$ of (3.1) depends on two separate time scales, the slow advective time scale $t$ and the fast time scale $\tau=t / \epsilon$ associated with the inertial waves. In addition, we assume that for $\epsilon \ll 1$ the solution has the asymptotic expansion,

$$
\boldsymbol{u}^{\epsilon}(\boldsymbol{x}, t)=\left.\boldsymbol{u}^{0}(\boldsymbol{x}, t, \tau)\right|_{\tau=t / \epsilon}+\left.\epsilon \boldsymbol{u}^{1}(\boldsymbol{x}, t, \tau)\right|_{\tau=t / \epsilon}+O\left(\epsilon^{2}\right),
$$

and it is also assumed that $\boldsymbol{u}^{1}(\boldsymbol{x}, t, \tau)=o(\tau)$, uniformly on $0 \leqslant \tau \leqslant T / \epsilon$, to guarantee the asymptotic validity of the expansion. The analysis of Embid and Majda then shows that to leading order in $\epsilon$ the solution $\boldsymbol{u}^{\epsilon}(\boldsymbol{x}, t)$ of (3.1) is given by,

$$
\boldsymbol{u}^{\epsilon}(\boldsymbol{x}, t)=\left.\boldsymbol{u}^{0}(\boldsymbol{x}, t, \tau)\right|_{\tau=t / \epsilon}+o(1)=e^{-t / \epsilon L_{F}} \boldsymbol{u}(\boldsymbol{x}, t)+o(1),
$$

where $\boldsymbol{u}(\boldsymbol{x}, t)$ solves a reduced equation obtained by averaging over the fast time variable $\tau$,

$$
\begin{array}{r}
\frac{\partial \boldsymbol{u}}{\partial t}+\lim _{T \rightarrow \infty} \frac{1}{T} \int_{0}^{T} e^{\tau L_{F}}\left[L_{S}\left(e^{-\tau L_{F}} \boldsymbol{u}\right)+B\left(e^{-\tau L_{F}} \boldsymbol{u}, e^{-\tau L_{F}} \boldsymbol{u}\right)-D\left(e^{-\tau L_{F}} \boldsymbol{u}\right)\right] \mathrm{d} \tau=0 \\
\left.\boldsymbol{u}(\boldsymbol{x}, t)\right|_{t=0}=\boldsymbol{u}_{0}(\boldsymbol{x}) .
\end{array}
$$

The fast wave averaging equation, (3.32), supersedes the slow dynamics equation, (3.13), whenever inertial waves are present. We also remark that the asymptotic analysis of Embid \& Majda (1998) can be justified with complete mathematical rigour via the technique of cancellation of oscillations developed in the important paper of Schochet (1994).

In practice it may be difficult to evaluate the limit over the fast variable $\tau$ in (3.32). However, this calculation can be performed in the case of periodic boundary conditions. In this case, the fast operator $L_{F}$ has an orthonormal basis of periodic eigenfunctions of the form,

$$
\boldsymbol{u}_{k}^{\alpha}(\boldsymbol{x})=\mathrm{e}^{\mathrm{i} k \cdot x} \boldsymbol{r}_{k}^{\alpha},
$$

where $\boldsymbol{k}=(k, l, m)$ is the wavenumber, $\alpha$ indicates whether the mode is slow $(\alpha=0)$ or fast $(\alpha= \pm 1)$, and $\boldsymbol{r}_{k}^{\alpha}=\left(\boldsymbol{v}_{k}^{\alpha}, \rho_{k}^{\alpha}\right)$. The associated purely imaginary eigenvalue is $\lambda_{k}^{\alpha}=\mathrm{i} \omega_{k}^{\alpha}$, where the frequency $\omega_{k}^{\alpha}$ is given by (3.9), namely $\omega_{k}^{ \pm 1}= \pm m /|\boldsymbol{k}|$ and $\omega_{k}^{0}=0$. The explicit form of these eigenfunctions is given in the Appendix. Next, we expand $\boldsymbol{u}(\boldsymbol{x}, t)$ in terms of the eigenfunctions of $L_{F}$,

$$
\boldsymbol{u}(\boldsymbol{x}, t)=\sum_{k} \sum_{\alpha=-1}^{1} \sigma_{k}^{\alpha}(t) \mathrm{e}^{\mathrm{i} k \cdot x} \boldsymbol{r}_{k}^{\alpha},
$$

and introduce this expansion into the fast wave averaging equation, (3.32). In order to evaluate the fast time averaging in (3.32) we observe that

$$
\mathrm{e}^{\tau L_{F}}\left(\mathrm{e}^{\mathrm{i} k \cdot x} \boldsymbol{r}_{k}^{\alpha}\right)=\mathrm{e}^{\mathrm{i} \tau \omega_{k}^{\alpha}} \mathrm{e}^{\mathrm{i} k \cdot x} \boldsymbol{r}_{k}^{\alpha},
$$


and also that

$$
\lim _{T \rightarrow \infty} \frac{1}{T} \int_{0}^{T} \mathrm{e}^{\mathrm{i} \omega \tau} \mathrm{d} \tau=\left\{\begin{array}{ll}
1 & \text { if } \omega=0 \\
0 & \text { if } \omega \neq 0
\end{array} .\right.
$$

With these observations we can evaluate all the terms in the limiting fast dynamics equation, (3.32), and conclude that the Fourier amplitudes $\sigma_{k}^{\alpha}(t)$ satisfy the system of differential equations,

$$
\frac{\mathrm{d} \sigma_{k}^{\alpha}}{\mathrm{d} t}+\sum_{R_{k}^{\alpha}} B_{\left(k^{\prime}, k^{\prime \prime}, k\right)}^{\left(\alpha^{\prime}, \alpha^{\prime \prime}, \alpha\right)} \sigma_{k^{\prime}}^{\alpha^{\prime}} \sigma_{k^{\prime \prime}}^{\alpha^{\prime \prime}}+\sum_{S_{k}^{\alpha}} L_{k}^{\left(\alpha^{\prime}, \alpha\right)} \sigma_{k}^{\alpha^{\prime}}=\sum_{S_{k}^{\alpha}} D_{k}^{\left(\alpha^{\prime}, \alpha\right)} \sigma_{k}^{\alpha^{\prime}} .
$$

The first sum in (3.37) comes from averaging the nonlinear advection term $B(\boldsymbol{u}, \boldsymbol{u})$ with summation over the set of three-wave resonant interactions $R_{k}^{\alpha}=\left\{\left(\boldsymbol{k}^{\prime}, \boldsymbol{k}^{\prime \prime}, \alpha^{\prime}, \alpha^{\prime \prime}\right) \mid \boldsymbol{k}^{\prime}+\boldsymbol{k}^{\prime \prime}=\boldsymbol{k}, \omega_{k^{\prime}}^{\alpha^{\prime}}+\omega_{k^{\prime \prime}}^{\alpha^{\prime \prime}}=\omega_{k}^{\alpha}\right\}$. The second and third sums come from averaging the slow operator $L_{S}$ and the diffusion operator $D$, respectively, with summation over the set $S_{k}^{\alpha}=\left\{\alpha^{\prime} \mid \omega_{k}^{\alpha^{\prime}}=\omega_{k}^{\alpha}\right\}$. Formulas for the interaction coefficients $B_{\left(k^{\prime}, k^{\prime \prime}, k\right)}^{\left(\alpha^{\prime}, \alpha^{\prime \prime}, \alpha\right)}, L_{k}^{\left(\alpha^{\prime}, \alpha\right)}$ and $D_{k}^{\left(\alpha^{\prime}, \alpha\right)}$ are included in the Appendix.

The fast dynamics equations for the Fourier amplitudes in (3.37) suggest that there is strong interaction of the fast and slow modes through three-waves interactions, via the quadratic interaction coefficients $B_{\left(k^{\prime}, k^{\prime \prime}, k\right)}^{\left(\alpha^{\prime}, \alpha^{\prime \prime}, \alpha\right)}$. However, it is remarkable that the dynamics of the slow modes $(\alpha=0)$ proceeds independent of the fast modes $(\alpha= \pm 1)$, making the system of slow and fast modes only weakly coupled. This is because all the interaction coefficients $B_{\left(k^{\prime}, k^{\prime \prime}, k\right)}^{( \pm 1, \pm 1,0)}$ corresponding to 'fast + fast $\rightarrow$ slow' interaction are always zero. The verification of this fact is given in the Appendix. Of course, the equation for the dynamics of the slow modes in (3.37) is nothing more than previously derived slow limiting dynamics equations, (3.13), recast in terms of the Fourier modes. Nevertheless, the slow modes influence the dynamics of the fast modes in (3.37) through 'fast + slow $\rightarrow$ fast' interactions. These conclusions mirror those previously derived by Embid \& Majda (1998) for the case of small Froude number.

A remarkable consequence of this weak coupling is that in the absence of dissipation there is conservation of energy for the slow and the fast modes separately. The reasoning, which was first provided by Embid \& Majda (1998) for the case of small Froude number, is reproduced below. First we observe that the solution $\boldsymbol{u}(\boldsymbol{x}, t)$ of the fast limiting dynamics, (3.32), has a unique orthogonal decomposition in terms of slow and a fast component,

$$
\boldsymbol{u}(\boldsymbol{x}, t)=\boldsymbol{u}^{S}(\boldsymbol{x}, t)+\boldsymbol{u}^{F}(\boldsymbol{x}, t) .
$$

In fact, $\boldsymbol{u}^{S}(\boldsymbol{x}, t)$ is given explicitly by (3.34) with $\alpha=0$ and $\boldsymbol{u}^{F}(\boldsymbol{x}, t)$ by (3.34) with $\alpha= \pm 1$. Next we substitute $\boldsymbol{u}(\boldsymbol{x}, t)$ into (3.31) and make use of (3.35) to conclude that to leading order in $\epsilon$ the solution $\boldsymbol{u}^{\epsilon}(\boldsymbol{x}, t)$ has the form,

$$
\boldsymbol{u}^{\epsilon}(\boldsymbol{x}, t)=\boldsymbol{u}^{S}(\boldsymbol{x}, t)+\mathrm{e}^{-t / \epsilon L_{F}} \boldsymbol{u}^{F}(\boldsymbol{x}, t)+o(1) .
$$

Moreover, since $L_{F}$ is a skew-Hermitian operator, then $\mathrm{e}^{t / \epsilon L_{F}}$ is an unitary operator, and since the eigenfunctions of $L_{F}$ are also an orthonormal basis for $\mathrm{e}^{t / \epsilon L_{F}}$ (see Lax 2002), we conclude that

$$
\|\boldsymbol{u}\|^{2}=\left\|\boldsymbol{u}^{S}\right\|^{2}+\left\|\mathrm{e}^{t / \epsilon L_{F}} \boldsymbol{u}^{F}\right\|^{2}=\left\|\boldsymbol{u}^{S}\right\|^{2}+\left\|\boldsymbol{u}^{F}\right\|^{2},
$$

where $\|\boldsymbol{u}\|^{2}=\int|\boldsymbol{v}|^{2}+\rho^{2} \mathrm{~d} v$ is twice the total energy (kinetic plus potential). In the absence of dissipation, (2.13) shows that the Boussinesq equations conserve energy, so that $\|\boldsymbol{u}\|^{2}$ is constant in time. On the other hand, the slow limiting dynamics equations 


\begin{tabular}{cccrrrrrrr} 
Run number & $R o$ & $F r$ & \multicolumn{1}{c}{$f$} & $N$ & $k_{f}$ & \multicolumn{1}{c}{$k_{d}$} & $\epsilon_{f}$ & $k_{\text {total }}$ & $T_{0}$ \\
1 & 1.0 & 1.0 & 7.0827 & 7.0827 & 3 & 3 & 1.0 & 256 & 20 \\
2 & 0.3 & 1.0 & 23.6091 & 7.0827 & 3 & 10.0 & 1.0 & 256 & 20 \\
3 & 0.2 & 1.0 & 35.4136 & 7.0827 & 3 & 15.0 & 1.0 & 256 & 20 \\
4 & 0.1 & 1.0 & 70.8273 & 7.0827 & 3 & 30.0 & 1.0 & 256 & 20 \\
5 & 0.08 & 1.0 & 88.5341 & 7.0827 & 3 & 37.5 & 1.0 & 256 & 20 \\
6 & 0.05 & 1.0 & 141.6546 & 7.0827 & 3 & 60.0 & 1.0 & 256 & 20 \\
7 & 0.01 & 1.0 & 708.2731 & 7.0827 & 3 & 300.0 & 1.0 & 256 & 60
\end{tabular}

TABLE 1. This figure tabulates the parameters used in the simulations at $256^{3}$ with low wavenumber forcing.

also conserve energy, so that $\left\|\boldsymbol{u}^{S}\right\|^{2}$ is also constant in time. Combining these two facts with (3.40), we conclude that $\left\|\boldsymbol{u}^{F}\right\|^{2}$ is constant in time, thus proving that the energies of $\boldsymbol{u}^{S}$ and $\boldsymbol{u}^{F}$ are constant separately. This important physical property of the fast limiting dynamics equations will be exploited later as a diagnostic tool in the numerical simulations.

Finally, we remark that the Fourier basis in (3.33) can be used to study the dependence of other related physical quantities on the slow and fast modes. For example, the leading term of the potential vorticity $q$ in (2.11) has the eigenfunction expansion

$$
q=\frac{\partial \rho}{\partial z}=\mathrm{i} \sum_{k} \sum_{\alpha=-1}^{1} m \rho_{k}^{\alpha} \sigma_{k}^{\alpha}(t) \mathrm{e}^{\mathrm{i} k \cdot x},
$$

where $\rho_{k}^{\alpha}$ is the fourth component of the vector $\boldsymbol{r}_{k}^{\alpha}$ in (3.33). Inspection of these vectors in (A 2) and (A 3) in the Appendix reveals that $\rho_{k}^{ \pm 1}=0$ and in consequence the $q$ is composed of slow modes even with the presence of fast inertial waves in the fast limiting dynamics. This fact can be used as the starting point to prove conservation of potential vorticity, in the weak sense and without dissipation, along the same lines developed in Embid \& Majda (1998). By contrast, the eigenfunction expansion of the vertical component of the vorticity $\omega$ is

$$
\omega=\frac{\partial v}{\partial x}-\frac{\partial u}{\partial y}=\mathrm{i} \sum_{k} \sum_{\alpha=-1}^{1}\left(k v_{k}^{\alpha}-l u_{k}^{\alpha}\right) \sigma_{k}^{\alpha}(t) \mathrm{e}^{\mathrm{i} k \cdot x},
$$

with $u_{k}^{\alpha}$ and $v_{k}^{\alpha}$ being the first and second components of $\boldsymbol{r}_{k}^{\alpha}$ in (3.33). Another inspection of (A 2) and (A 3) in the Appendix reveals that $k v_{k}^{0}-l u_{k}^{0}=0$ and we conclude that $\omega$ is exclusively composed of fast modes.

\section{Numerical simulations}

The goal of this section is to see if key attributes of the $F r \approx 1, R o \rightarrow 0$ limiting dynamics can be reproduced in numerical simulations that use low wavenumber white noise forcing. The three aspects we examine are: (i) the columnar structure, (ii) the time evolution of the ratio of the $E_{R o \rightarrow 0}$ (slow) total energy to the total energy, $E$, and (iii) the time evolution of the ratio of the $Q_{R o \rightarrow 0}$ (slow) potential enstrophy to the total potential enstrophy, $Q$.

For all our simulations, detailed in table 1, we use the triply periodic, pseudospectral LANL/Sandia direct numerical simulation code that solves (2.1) and (2.2) 
in rectangular domains $\left(2 \mathrm{D}[0,1]^{2}\right.$ or $\left.3 \mathrm{D}[0,1]^{3}\right)$ with a pseudo-spectral method or fourth order finite differences and a RK4 time stepping scheme. The code allows for an arbitrary number of passive scalars and arbitrary aspect ratio grids. The code uses MPI for its parallelisation along with a 3D domain decomposition (allowing for slab decomposition, pencil decomposition or cube decomposition). Since its inception, it has been designed for performance on massively parallel computers and has excellent scalability, which has been demonstrated on up to 18000 processors running problems as large as $4096^{3}$ (64B grid points). All diagnostics and associated I/O are also implemented with fully scalable algorithms. The parallel fast Fourier transform (FFT) at the core of the model is one of the fastest available. It uses a custom data transpose algorithm which overlaps inter-process communication with on-processor data rearrangement allowing the code to rely exclusively on stride 1, on-processor FFTs (for which the code uses the fastest Fourier transform in the West (FFTW)).

For the code's configuration we turn to a paper by Smith \& Waleffe (2002). In that work they not only studied the generation of large, slow scales in rotating and stratified flow but they also found that for strongly stratified flows they recovered the sheet-like structures described when vertically sheared horizontal (VSH) dynamics dominates which was discussed Embid \& Majda (1998), Riley \& Lelong (2000), Riley \& deBruynKops (2003), Babin et al. (1997). In the spirit of those simulations, we examine some aspects of the low Rossby number limit by using a similar simulation set-up. The principal difference between the code set-up of Smith \& Waleffe (2002) and this work is that instead of using high wavenumber white noise forcing we use low wavenumber white noise forcing which can be understood by considering the Rossby deformation radius, $L_{d}$,

$$
L_{d}=\frac{N}{f} L_{f} \quad \text { or } \quad k_{d}=\frac{f}{N} k_{f} .
$$

where $k_{f}$ is the peak wavenumber of the forcing and $k_{d}$ is the wavenumber of the deformation radius. This equation shows that the important horizontal length scales described by $k_{d}$ increase with increasing rotation rate assuming $N$ is held fixed (see table 1). Because of limited resolution we choose $k_{f}=3$ for all the simulations presented in this paper.

For the sake of completeness, we outline some of the details of the Smith \& Waleffe (2002) code configuration. First, in order to reduce the effects of viscosity in the intermediate range of scales, a hyperviscosity replaces the Laplacian dissipation used in (2.1). The momentum dissipation is replaced by $(-1)^{p+1} v_{h}\left(\nabla^{2}\right)^{p} \boldsymbol{v}$ and the buoyancy by $(-1)^{p+1} \kappa_{h}\left(\nabla^{2}\right)^{p} \rho$, where $p=8$ for all the simulations presented in this paper. The hyperviscosity, $v_{h}$, is

$$
v_{h}=2.5\left(\frac{E\left(k_{m}, t\right)}{k_{m}}\right)^{1 / 2} k_{m}^{2-2 p}
$$

as in Chasnov (1994), where $k_{m}$ is the highest available wavenumber and $E\left(k_{m}, t\right)$ is the kinetic energy in the highest available wavenumber shell. The hyperdiffusivity used for the buoyancy equation is similar. The random forcing spectrum $F(k)$ is Gaussian with a standard deviation $s=1$ and energy input rate $\epsilon_{f}=1$ given by

$$
F(k)=\epsilon_{f} \frac{\exp \left(-0.5\left(k-k_{f}\right)^{2} / s^{2}\right)}{(2 \pi)^{1 / 2} s} .
$$



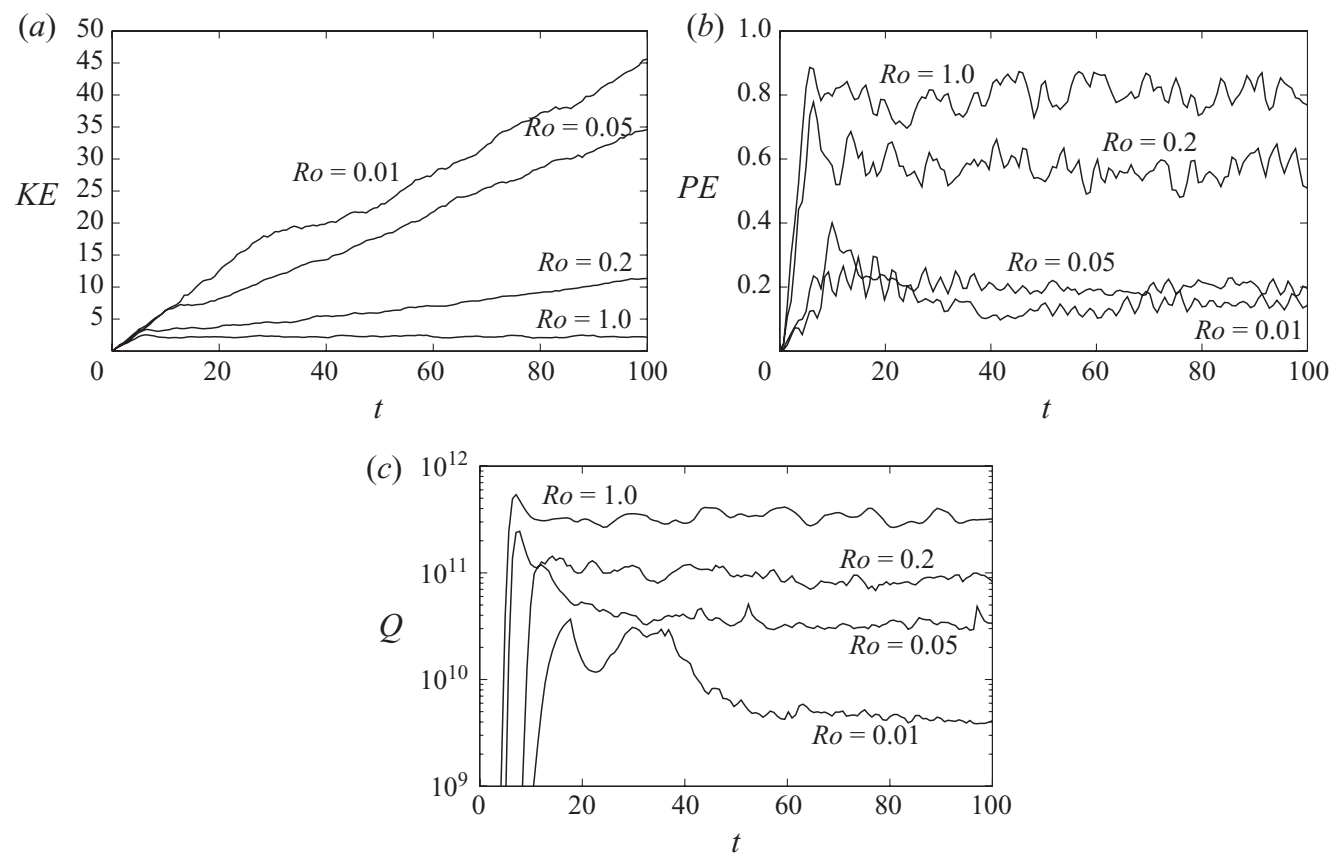

FIGURE 1. To characterise the numerical simulations, this figure shows the time evolution of $(a)$ kinetic energy, $(b)$ potential energy and (c) potential enstrophy for selected Rossby numbers and $F r=1$. Energy, potential enstrophy and time have been non-dimensionalised using the scales in (4.5). The kinetic energy grows in time due to the momentum forcing. After a spin-up time, comparison of the different runs shows that the smaller the Rossby number the larger the magnitude of the kinetic energy, and the smaller the magnitude of the potential energy. The order of magnitude of the potential enstrophy also decreases with decreasing Rossby number. For all the simulations except $R o=1$ columns appear that span the depth of the fluid. These columns are dynamic and remain columnar for the duration of the simulation.

In like manner we also use Rossby and Froude numbers based on the energy input rate $\epsilon_{f}$ and the peak wavenumber $k_{f}$ of the forcing,

$$
F r=\frac{\left(\epsilon_{f}\left(2 \pi k_{f}\right)^{2}\right)^{1 / 3}}{N} \text { and } R o=\frac{\left(\epsilon_{f}\left(2 \pi k_{f}\right)^{2}\right)^{1 / 3}}{f} .
$$

The characteristic scales for time, energy and potential enstrophy are

$$
\mathscr{T}=\left(\epsilon_{f}\left(2 \pi k_{f}\right)^{2}\right)^{-1 / 3}, \quad \mathscr{E}=\left(\epsilon_{f}\left(2 \pi k_{f}\right)^{-1}\right)^{2 / 3} \text { and } \mathscr{Q}=\rho_{o}^{2}\left(\epsilon_{f}\left(2 \pi k_{f}\right)^{5}\right)^{2 / 3} \text {. }
$$

These scales are used throughout the numerical section to non-dimensionalise time, energy and potential enstrophy. The factors of $2 \pi$ appear in the above expressions because the code has a domain of $[0,1]^{3}$.

Each run is spun up from zero and forced throughout by the low wavenumber white noise forcing described by (4.3). The parameters $N$ and $f$ remain fixed throughout the simulation. For runs where $(R o<1)$ columnar structures form during the spin up period. The columns are dynamic and retain their basic columnar structure in the quantities $\bar{u}^{z}$ and $\bar{u}^{x}$ throughout the simulation (see figure 3 ).

The evolution of the kinetic energy, potential energy and potential enstrophy, nondimensionalised using the scales in (4.5), for selected Rossby numbers is shown in figure 1. Because the flow is forced in the momentum equations the kinetic energy 

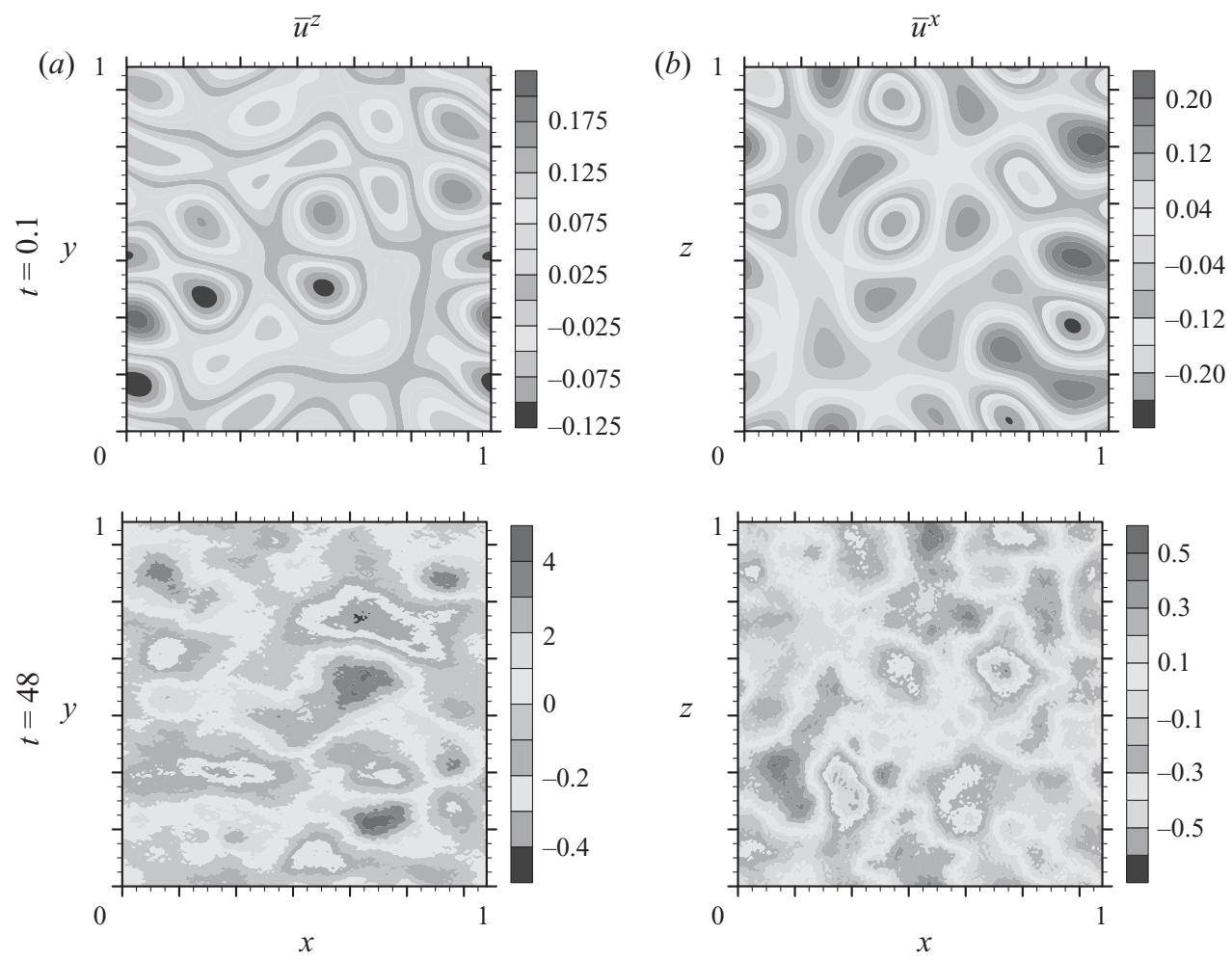

FIGURE 2. This figure shows vertical $(a)$ and horizontal $(b)$ averages of the horizontal component of the velocities for $R o=1$ and $F r=1$ simulations of the full Boussinesq equations. Patterns form on length scales consistent with the low wavenumber forcing of $k_{f}=3$ but no columns form.

increases with time. After a spin-up time, comparison of the different runs shows that the smaller the Rossby number the larger the magnitude of the kinetic energy, and the smaller the magnitude of the potential energy. The order of magnitude of the potential enstrophy also decreases with decreasing Rossby number.

\subsection{Columnar Taylor-Proudman flows}

The classical Taylor-Proudman result is that for constant density flow in geostrophic and hydrostatic balance the vertical derivatives of the horizontal and vertical velocities are zero, creating columnar flows. Our theory, described by the projection operator, (3.17), generalises the Taylor-Proudman theory to the case when the density is not constant and the flow is not in hydrostatic balance. We examine the flow characteristics at different Rossby numbers to see if Taylor-Proudman flows appear. First we define two averages of $u$, the $x$ component of the horizontal velocity,

$$
\bar{u}^{z}=\int_{L} u\left(x, y, z, t_{o}\right) 2 \pi \mathrm{d} z, \quad \text { and } \bar{u}^{x}=\int_{L} u\left(x, y, z, t_{o}\right) 2 \pi \mathrm{d} x,
$$

where $L=1$ and $t_{o}$ is any time after the spin-up time. Figures 2 and 3 show contour plots of $\bar{u}^{z}$, which we used to identify large-scale horizontal structures, and contour plots of $\bar{u}^{x}$, which we use to identify large-scale vertical structures. Plot $(a)$ of each figure shows contours of $\bar{u}^{z}$ while plot (b) shows contours of $\bar{u}^{x}$. Figure 2 shows the 

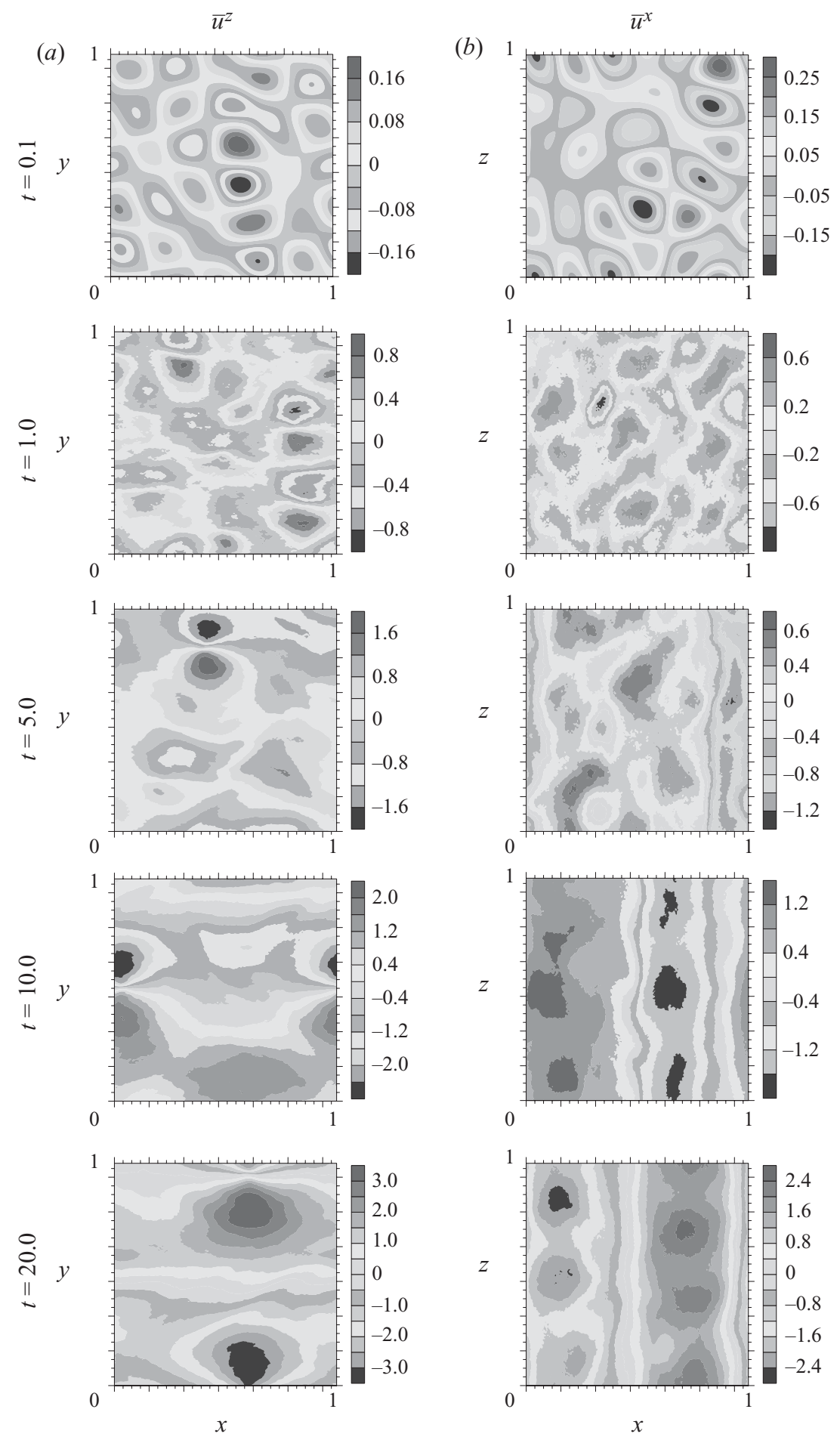

FIgURE 3. This figure shows vertical $(a)$ and horizontal $(b)$ averages of the horizontal component of the velocities for $R o=0.2$ and $F r=1$ simulations of the full Boussinesq equations. By time $t=10$ columnar structures are beginning to form. At $t=20$ the dominant columnar structures have formed and remain columnar through the rest of the simulation. 
case when $R o=1$ (not small). The main structures of this flow are consistent with $k_{f}=3$ forcing scale but never become columnar flows. On the right of figure 3 , for the case of a smaller Rossby number, $R o=0.2$, the contours reveal a low wavenumber vortical structure. That this is a columnar structure becomes evident by examining the lower right panel of figure 3 , which shows the contour plot of $\bar{u}^{x}$. There is no similar columnar structure for the $R o=1$ run. Therefore, even with low wavenumber white noise forcing columnar Taylor-Proudman flows spontaneously form if the Rossby number is low enough.

Though much is known about the formation and instability of vortices in rotating and stratified flow, this is the first study that we know of where a constant-in-time white noise forcing creates columnar structures that remain columnar throughout the length of the simulation.

Other studies that discuss the formation of columnar structures in rotating turbulence (with no buoyancy) can be found in Davidson, Staplehurst \& Dalziel (2006), Staplehurst, Davidson \& Dalziel (2008), Sreenivasan \& Davidson (2008). These are studies about the creation and evolution of vortices from an initial condition, unlike this work which has a constant-in-time white noise forcing, and they do not consider stratification, while this work considers only weak stratification. Despite this, the mechanisms they found for columnar vortex formation are relevant to this study. In Davidson et al. (2006), Staplehurst et al. (2008), they begin by considering an initial blob of fluid in a rotating environment. They find that when the rotation is strong enough linear wave energy propagation is biased along the axes of rotation and that when the columnar vortex appears it remains contained in the cylinder that circumscribes the initial blob. When the rotation is weak a centrifugal bursting phenomenon prevents any columnar vortices from forming. We can see evidence for this in figure 3 as the wavenumber 3 structures elongate and finally form columnar structures. There are two classical laboratory studies of the formation of columnar vortices and both employ a constant-in-time forcing: the original laboratory studies by Taylor (1921) and Davies (1972). Both investigators studied the dynamics associated with the slow, steady, horizontal motion of a solid obstacle through a fluid rotating about a vertical axis. One main difference between the studies is that Davies (1972) included stratification and Taylor (1921) did not. Both investigators find the formation of columnar structures above the moving topographic feature. While there are considerable differences in the kinds of columnar vortices that form, when they did form there was no mention of them becoming unstable.

There is also a large body of literature on the stability of columnar vortices in rotating and stratified flow. We restrict this discussion to key work related to this study (strong rotation and weak stratification, i.e. non-hydrostatic). We first consider the work of Potylitsin \& Peltier (1998) in which they use linear stability analysis to study the stability of columnar vortices to three-dimensional perturbations. They use two initial distributions of vorticity: Kelvin-Helmholtz-generated vortices in shear and Kida-like vortices in strain. Their conclusion is that an isolated anti-cyclonic vortex column is strongly destabilised by small values of the background rotation, while rapid rotation stabilises both cyclonic and anti-cyclonic initial conditions. They explain this phenomenon using the Taylor-Proudman theorem. They also discuss the details of the stability of anti-cyclonic vortices but since all our columnar vortices are cyclonic we will not describe their other results here. The next related paper is Potylitsin \& Peltier (2003) where they use direct numerical simulations to study the evolution of Kelvin-Helmholtz-generated columnar vortices and verify their previous results that strong rotation stabilises the columnar vortices. The last work we will 

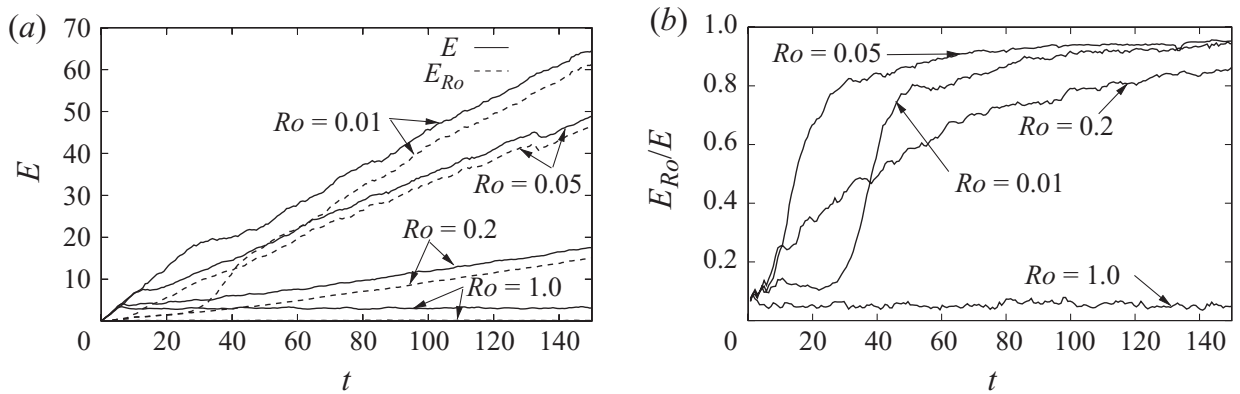

Figure 4. Plot ( $a$ ) shows the total energy, $E$ (solid line) and the slow total energy, $E_{R o}$ (dashed line). The total energy and the slow total energy appear to be on parallel trajectories in time. This is explored more fully in $(b)$ which shows the time evolution of the ratio $E_{R o} / E$. As the Rossby number decreases, a larger fraction of the total energy is slow. Furthermore, the low Rossby number runs appear to be approaching a constant at late times.

examine is Otheguy, Chomaz \& Billant (2006). This work also uses a linear stability analysis but begins with an initial condition of co-rotating vortices. When there is no stratification, they find, as Potylitsin \& Peltier (1998), that the columnar vortex is elliptic unstable and that stronger stratification causes a zig-zag instability. This work is not directly applicable to our work because it studies the evolution of an initial condition of co-rotating vortices which our simulations do not show but it shows that the evolution of columnar vortices depends on the rotation, stratification, initial conditions and forcing.

\subsection{Ratio of slow energy to total energy}

Our theory states that in the absence of viscosity the total energy is composed of both fast and slow dynamics but that the ratio of the $R o \rightarrow 0$ (slow) total energy, $E_{R o}$, to the total energy, $E$, should go to a constant (cf. the discussion following (3.40))

$$
\frac{E_{R o}}{E} \rightarrow C \text { for } \quad v=0 .
$$

In this section we examine the time evolution of $E_{R o} / E$ of our numerical simulations for varying Rossby numbers. The total energy, $E$, is given by

$$
E=\int_{V} \frac{1}{2}\left(|\boldsymbol{v}|^{2}+\rho^{2}\right)(2 \pi)^{3} \mathrm{~d} v,
$$

where $V=1$. The $R o \rightarrow 0$ (slow) component is computed by projecting the full solution vector, $(u, v, w, \rho)$ onto the null space of the fast operator using (3.17). The total energy from the slow variables is then computed by combining the slow horizontal kinetic energy described by (3.22) and the total vertical energy described in (3.27),

$$
E_{R o}=\int_{A} \frac{1}{2}\left(\left|\boldsymbol{v}_{H}\right|^{2}+w^{2}+\langle\rho\rangle_{z}^{2}\right)(2 \pi)^{2} \mathrm{~d} a,
$$

where $A=1$ and, as mentioned above, all the variables are the result of projecting the full solution vector onto the null space of the fast operator. Figure 4(a) shows the evolution of both $E$ (solid line) and $E_{R o}$ (dashed line) with time. At fixed time, both the slow total energy and the total energy have larger amplitudes as the Rossby number decreases. The figure also shows that the total energy and the slow total energy appear to maintain a similar ratio as time increases. This is explored more fully in figure $4(b)$ where we plot the ratio $E_{R o} / E$. We find that when $R o=1$ the ratio 


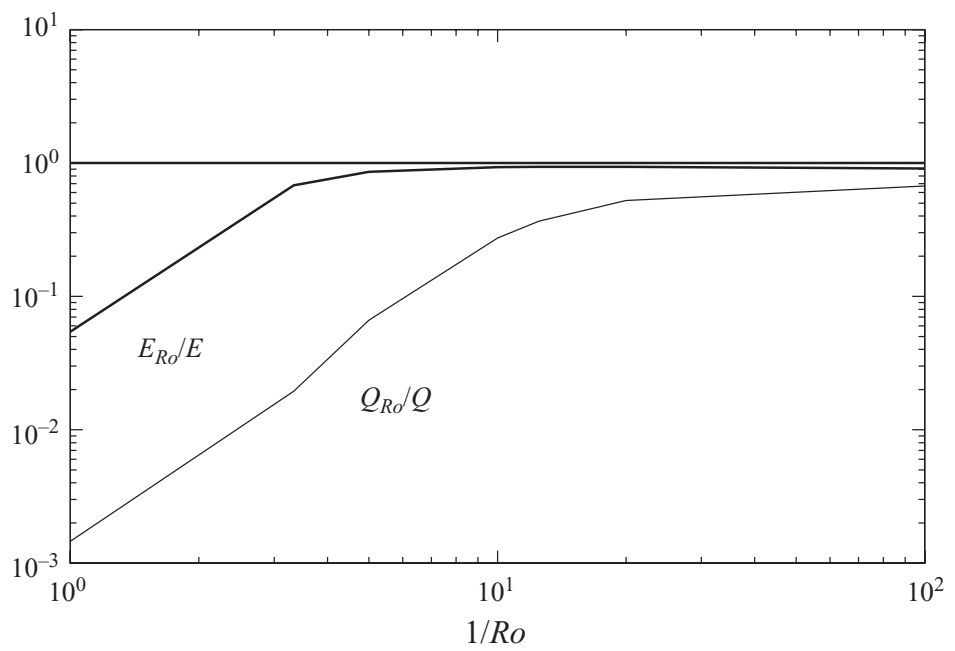

FIGURE 5. This figure shows the dependence of the time averaged quantities $\bar{E}_{R o}^{T} / \bar{E}^{T}$ and $\bar{Q}_{R o}^{T} / \bar{Q}^{T}$ on $R o$. As the Rossby number decreases the simulations show that a larger fraction of the total energy and potential enstrophy is slow.

$E_{R o} / E$ maintains approximately the same percentage of slow to total energy, but that the slow component is a small fraction of the total. As we decrease the Rossby number the time evolution of $E_{R o} / E$ shows a gradual increase in the percentage of energy that is slow relative to the total. For the smallest Rossby numbers we ran, the ratio quickly increases to a value where a substantial fraction of the total energy is its slow component and then gradually increases towards a constant close to 1 . While $E_{R o} / E$ is not a constant in time, it is nearly so for the smallest Rossby numbers. This suggests that even when there is dissipation and forcing in the system there is a rapid initial adjustment of $E_{R o} / E$ towards a value that indicates a significant fraction of the total energy is slow, then a gradual increase towards a constant close to 1.

To examine the explicit dependence of $E_{R o} / E$ on Rossby number, we compute its time average, $\bar{E}_{R o}^{T} / \bar{E}^{T}$, where we compute the average using

$$
\bar{X}^{T}=\frac{1}{T_{F}-T_{0}} \sum_{i=T_{0}}^{T_{F}} X_{i} \mathrm{~d} t_{i},
$$

where $T_{0}$ is a time immediately after spin up and $T_{F}$ is the last time available from the simulation. This quantity is shown in figure 5 where we see the trend that as the Rossby number decreases, the ratio of slow total energy to total energy is approaching a constant and that the constant is close to 1. This implies that at the low Rossby numbers most of the total energy in these simulations is slow.

\subsection{Ratio of slow potential enstrophy to total potential enstrophy}

Our theory shows that in the limit of $R o \rightarrow 0$ and $F r=O(1)$, the potential enstrophy contains only slow dynamics (see the discussion below (3.41)). Stated another way, the ratio of the slow potential enstrophy, $Q_{R o}$, to the total potential enstrophy, $Q$, goes to 1 ,

$$
\frac{Q_{R o}}{Q} \rightarrow 1 \quad \text { for } v=0
$$



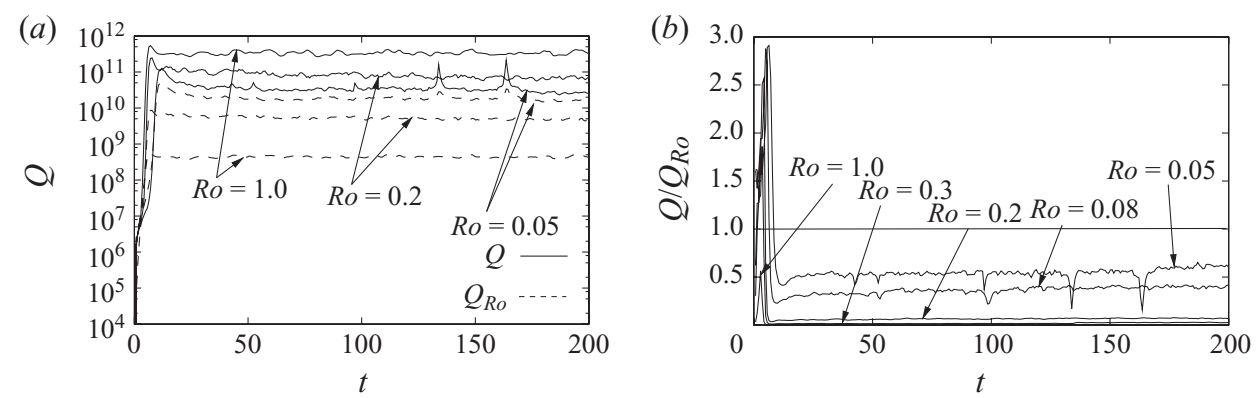

Figure 6. Plot ( $a$ ) shows the time evolution of the potential enstrophy (solid line) and the slow potential enstrophy (dashed line). As the Rossby number decreases the curves that represent the slow potential enstrophy and the total potential enstrophy converge towards each other. This is explored more fully in $(b)$ which shows the time evolution of the ratio $Q_{R o} / Q$. For the lowest Rossby number, $R o=0.01$, the magnitude of the ratio starts to decrease after about $T=30$ indicating that the slow component of the potential enstrophy decreases. However, at that Rossby number the simulations are approaching the limit of what $256^{3}$ simulations can resolve. For the other Rossby numbers, as Ro decreases this ratio approaches 1, showing that in the limit of fast rotation and order one stratification a large fraction of their potential enstrophy is its slow component - even with white noise forcing.

Here we examine the time evolution of $Q_{R o} / Q$ for our numerical simulations for a sequence of decreasing Rossby numbers. We compute the total potential enstrophy as,

$$
Q=\int_{V} \frac{1}{2} q^{2} \mathrm{~d} v,
$$

where $V=(2 \pi)^{3}$ and $q$ is the non-dimensional potential vorticity described by (2.11). The $R o \rightarrow 0$ component of potential enstrophy is computed by projecting the full solution vector onto the null space of the fast operator, (3.17). However, since the projection operator does not affect $\rho$, the $R o \rightarrow 0$ component of the potential enstrophy can be computed by

$$
Q_{R o}=\int_{V} \frac{1}{2}\left(\frac{\partial \rho}{\partial z}\right)^{2} \mathrm{~d} v .
$$

The panel on the left of figure 6 compares the time evolution of $Q$ (solid lines) with the time evolution of $Q_{R o}$ (dashed lines). As the Rossby number decreases, the gap between the solid and dashed lines decreases, indicating that the total enstrophy's composition has a larger slow component. This is explored further in figure 6(b) where we show the ratio $Q_{R o} / Q$. As the Rossby number tends to smaller values the slow component of the potential enstrophy becomes a larger fraction of the total potential enstrophy. This suggests that even with low wavenumber white noise forcing the potential enstrophy of this limit is dominated by its slow component. We also note that as the Rossby number decreases the vorticity is expected to have a larger fast component (see §3.3) which means the potential enstrophy defined by (4.12) can be replaced with (4.13).

Finally, to see the dependence of the ratio $Q_{R o} / Q$ on the Rossby number, we plot the quantity $\bar{Q}_{R o}^{T} / \bar{Q}^{T}$ where the averages are computed using (4.10) and are shown in figure 5. This figure shows that $Q_{R o} / Q \rightarrow 1$ as $R o$ decreases, the ratio is approaching 1 but more slowly in Rossby number than that of the energy. Dimensionally this means the globally integrated potential enstrophy is dominated by the vertical gradient 
of the buoyancy times the Coriolis parameter while both the vorticity times the Brunt-Väisäla frequency and the nonlinear terms have lesser influence.

\section{Summary}

We have examined the fast rotation limit of the rotating and stratified Boussinesq equations using the framework of Embid \& Majda (1998). We have shown that to leading order, the dynamics is composed of both fast and slow components but that the slow dynamics evolves independent of the fast. We have also derived new equations for the slow dynamics. These include the two-dimensional Navier-Stokes equations for the slow horizontal velocity, a forced advection-=diffusion equation for the vertically averaged vertical velocity, making the slow dynamics non-hydrostatic, and an equation for the buoyancy which is the only quantity to retain its threedimensional character. In the absence of viscosity and diffusivity these new sets of equations conserve a horizontal kinetic energy and vertical vorticity, along with a new conserved quantity that describes dynamics between the vertical kinetic energy and the buoyancy. The leading order total energy contains both fast and slow dynamics, though their ratio is conserved. The potential energy is found, to leading order, to contain only slow dynamics.

Our numerical simulations, which used low wavenumber white noise forcing in the momentum equations, reveal the emergence of Taylor-Proudman flows as the Rossby number decreases. They also support the theory that the ratio of the slow to total energy goes to a constant as the Rossby number is decreased and that the constant is close to 1 . In addition to the energy we also examined the ratio of the slow potential enstrophy to the total potential enstrophy. The smallest Rossby number we examined, $R o=0.01$, was at the limit of the scales that could be resolved at this resolution. However, the other Rossby numbers showed the trend that the ratio of the slow potential enstrophy to total potential enstrophy also approaches a constant and that constant trends towards 1 . These numerical simulations indicate that some of the aspects dynamics derived in this paper exist even in the presence of white noise forcing and hyperviscosity.

\section{Appendix}

\section{A.1. Analysis of the fast operator}

The fast operator $L_{F}$ is defined on the Hilbert space $X$ of $2 \pi$-periodic squareintegrable vector fields $\boldsymbol{u}=(\boldsymbol{v}, \rho)$ that are divergence free, $\boldsymbol{\nabla} \cdot \boldsymbol{v}=0$. In the space $X$ the eigenfunctions of $L_{F}$ are given by Fourier modes of the form $\boldsymbol{u}_{k}(\boldsymbol{x})=\mathrm{e}^{\mathrm{i} k \cdot x} \boldsymbol{r}_{k}$, where $\boldsymbol{k}=(k, l, m)$ is the wave number and $\boldsymbol{r}_{k}=\left(\boldsymbol{v}_{k}, \rho_{k}\right)$ is a fixed vector. The divergence free condition reduces to the algebraic constraint $\boldsymbol{v}_{k} \cdot \boldsymbol{k}=0$. In terms of the Fourier eigenmode $\boldsymbol{u}_{k}(\boldsymbol{x})$ the eigenvalue equation $L_{F} \boldsymbol{u}_{k}=\lambda_{k} \boldsymbol{u}_{k}$ reduces to the algebraic eigenvalue problem $L_{F}(\boldsymbol{k}) \boldsymbol{r}_{k}=\lambda_{k} \boldsymbol{r}_{k}$, where the matrix symbol $L_{F}(\boldsymbol{k})$ is given, for $\boldsymbol{k} \neq \mathbf{0}$ and $\boldsymbol{k}=\mathbf{0}$, respectively, by

$$
L_{F}(\boldsymbol{k})=\frac{1}{|\boldsymbol{k}|^{2}}\left(\begin{array}{cccc}
-k l & -\left(l^{2}+m^{2}\right) & 0 & 0 \\
k^{2}+m^{2} & k l & 0 & 0 \\
-l m & k m & 0 & 0 \\
0 & 0 & 0 & 0
\end{array}\right), \quad L_{F}(\mathbf{0})=\left(\begin{array}{cccc}
0 & -1 & 0 & 0 \\
1 & 0 & 0 & 0 \\
0 & 0 & 0 & 0 \\
0 & 0 & 0 & 0
\end{array}\right),
$$


with $|\boldsymbol{k}|^{2}=k^{2}+l^{2}+m^{2}$. The algebraic eigenvalue problem has four purely imaginary eigenvalues, $\lambda_{k}=\mathrm{i} \omega_{k}^{\alpha}$, with $\omega_{k}^{ \pm 1}= \pm \frac{m}{k \mid}$ corresponding to fast inertial modes and the double eigenvalue $\omega_{k}^{0}=0$ corresponding to the slow modes. The associated eigenvectors are given as follows. If $\left|\boldsymbol{k}_{H}\right| \neq 0$ there are three eigenvectors,

$$
\boldsymbol{r}_{k}^{1}=\frac{1}{\sqrt{2}\left|\boldsymbol{k}_{H}\right||\boldsymbol{k}|}\left(\begin{array}{c}
-l|\boldsymbol{k}|+\mathrm{i} k m \\
k|\boldsymbol{k}|+\mathrm{i} l m \\
-\mathrm{i}\left|\boldsymbol{k}_{H}\right|^{2} \\
0
\end{array}\right), \quad \boldsymbol{r}_{k}^{-1}=\frac{1}{\sqrt{2}\left|\boldsymbol{k}_{H}\right||\boldsymbol{k}|}\left(\begin{array}{c}
l|\boldsymbol{k}|-\mathrm{i} k m \\
-k|\boldsymbol{k}|-\mathrm{i} l m \\
\mathrm{i}-\left.\boldsymbol{k}_{H}\right|^{2} \\
0
\end{array}\right), \quad \boldsymbol{r}_{k}^{0}=\left(\begin{array}{l}
0 \\
0 \\
0 \\
1
\end{array}\right) \text {. }
$$

The fourth eigenvector does not satisfy the incompressibility constraint $\boldsymbol{v}_{k} \cdot \boldsymbol{k}=0$. If $\left|\boldsymbol{k}_{H}\right|=0$ but $|\boldsymbol{k}| \neq 0$, then $\omega_{k}^{ \pm 1}= \pm 1, \omega_{k}^{0}=0$, and there are four eigenvectors,

$$
\boldsymbol{r}_{k}^{1}=\frac{1}{\sqrt{2}}\left(\begin{array}{c}
1 \\
-\mathrm{i} \\
0 \\
0
\end{array}\right), \quad \boldsymbol{r}_{k}^{-1}=\frac{1}{\sqrt{2}}\left(\begin{array}{l}
1 \\
\mathrm{i} \\
0 \\
0
\end{array}\right), \quad \boldsymbol{r}_{k}^{0}=\left(\begin{array}{l}
0 \\
0 \\
0 \\
1
\end{array}\right), \quad \tilde{\boldsymbol{r}}_{k}^{0}=\left(\begin{array}{l}
0 \\
0 \\
1 \\
0
\end{array}\right),
$$

but the fourth eigenvector, $\tilde{\boldsymbol{r}}_{k}^{0}$, violates the incompressibility constraint. Finally, if $|\boldsymbol{k}|=0$ then there are two fast modes associated with $\omega_{0}^{ \pm 1}= \pm 1$ and two slow modes associated with $\omega_{0}^{0}=0$, with the four eigenvectors in (A 3) now satisfying the incompressibility constraint. Notice that the eigenfunctions are normalised and satisfy the symmetry condition $\overline{\boldsymbol{r}_{k}^{\alpha}}=\boldsymbol{r}_{-k}^{-\alpha}$, where the bar stands for complex conjugation. For this reason, we require that the amplitudes $\sigma_{k}^{\alpha}(t)$ in (3.34) satisfy the condition $\overline{\sigma_{k}^{\alpha}}=\sigma_{-k}^{-\alpha}$ to ensure that $\boldsymbol{u}(\boldsymbol{x}, t)$ in (3.34) is real valued.

\section{A.2. Formulas for the interaction coefficients}

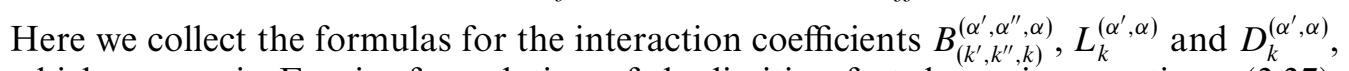
which appear in Fourier formulation of the limiting fast dynamics equations, (3.37). The quadratic interaction coefficient $B_{\left(k^{\prime}, k^{\prime \prime}, k\right)}^{\left(\alpha^{\prime}, \alpha^{\prime \prime}\right)}$ is given by

$$
B_{\left(k^{\prime}, k^{\prime \prime}, k\right)}^{\left(\alpha^{\prime}, \alpha^{\prime \prime}, \alpha\right)}=\frac{\mathrm{i}}{2}\left[\left(\boldsymbol{v}_{k^{\prime}}^{\alpha^{\prime}} \cdot \boldsymbol{k}^{\prime \prime}\right) \boldsymbol{r}_{k^{\prime \prime}}^{\alpha^{\prime \prime}}+\left(\boldsymbol{v}_{k^{\prime \prime}}^{\alpha^{\prime \prime}} \cdot \boldsymbol{k}^{\prime}\right) \boldsymbol{r}_{k^{\prime}}^{\alpha^{\prime}}\right] \cdot \overline{\boldsymbol{r}_{k}^{\alpha}}
$$

With this formula we can verify the claim that the fast limiting dynamics equations for the slow modes is independent of the fast modes, i.e. the quadratic interaction coefficients corresponding to 'fast + fast $\rightarrow$ slow' are zero. Because the formula for the quadratic interaction coefficient in (A 4) is invariant under the permutation of $\alpha^{\prime}$ and $\alpha^{\prime \prime}$, and $\boldsymbol{k}^{\prime}$ and $\boldsymbol{k}^{\prime \prime}$, it is sufficient to check that $B_{\left(k^{\prime}, k^{\prime \prime}, k\right)}^{(-1,1,0)}$ is zero. The three-wave resonance equations for this case is

$$
\boldsymbol{k}^{\prime}+\boldsymbol{k}^{\prime \prime}=\boldsymbol{k}, \quad \frac{m^{\prime}}{\left|\boldsymbol{k}^{\prime}\right|}-\frac{m^{\prime \prime}}{\left|\boldsymbol{k}^{\prime \prime}\right|}=0 .
$$

There are three cases to consider. First, if $\left|\boldsymbol{k}_{H}\right| \neq 0$ then $\boldsymbol{r}_{k}^{0}$ in (A 2) is orthogonal to $\boldsymbol{r}_{k}^{ \pm 1}$ in both (A 2) and (A 3), and $B_{\left(k^{\prime}, k^{\prime \prime}, k\right)}^{(-1,1,0)}$ is zero. Second, if $\left|\boldsymbol{k}_{H}\right|=0$ but $|\boldsymbol{k}| \neq 0$ then $\boldsymbol{r}_{k}^{0}$ in (A 3) coincides with $\boldsymbol{r}_{k}^{0}$ in the previous case and the quadratic coefficient is again zero. Finally, if $|\boldsymbol{k}|=0$ then there $\boldsymbol{r}_{k}^{\alpha}$ in (A 3) is either $\boldsymbol{r}_{k}^{0}$ or $\tilde{\boldsymbol{r}}_{k}^{0}$. In this case $\boldsymbol{k}^{\prime}=-\boldsymbol{k}^{\prime \prime}$ and, by symmetry, $\boldsymbol{r}_{-k^{\prime}}^{-1}=\overline{\boldsymbol{r}_{k^{\prime}}}$. Direct calculation then shows that the third component 
of $\left(\boldsymbol{v}_{k^{\prime}}^{1} \cdot\left(-\boldsymbol{k}^{\prime}\right)\right) \overline{\boldsymbol{r}_{k^{\prime}}^{1}}+\left(\overline{\boldsymbol{v}_{k^{\prime}}^{1}} \cdot \boldsymbol{k}^{\prime}\right) \boldsymbol{r}_{k^{\prime}}^{1}$ in (A 4) is given by

$$
\begin{aligned}
\left(\boldsymbol{v}_{k^{\prime}}^{1} \cdot\left(-\boldsymbol{k}^{\prime}\right)\right) \overline{w_{k^{\prime}}^{1}}+\left(\overline{\boldsymbol{v}_{k^{\prime}}^{1}} \cdot \boldsymbol{k}^{\prime}\right) w_{k^{\prime}}^{1} & =\mathrm{i}\left|\boldsymbol{k}_{H}^{\prime}\right|^{2}\left(\boldsymbol{v}_{k^{\prime}}^{1}+\overline{\boldsymbol{v}_{k^{\prime}}^{1}}\right) \cdot \boldsymbol{k}^{\prime} \\
& =2 \mathrm{i}\left|\boldsymbol{k}_{H}^{\prime}\right|^{2}\left(-l^{\prime}\left|\boldsymbol{k}^{\prime}\right|, k^{\prime}\left|\boldsymbol{k}^{\prime}\right|, 0\right) \cdot\left(k^{\prime}, l^{\prime}, m^{\prime}\right)=0,
\end{aligned}
$$

and this implies that the dot product with either $\boldsymbol{r}_{k}^{0}$ or $\tilde{\boldsymbol{r}}_{k}^{0}$ for the quadratic interaction coefficient in (A 4$)$ is again zero. This proves that $B_{\left(k^{\prime}, k^{\prime \prime}, k\right)}^{(-1,1,0)}$ is always zero.

Next, the linear interaction coefficient $L_{k}^{\left(\alpha^{\prime}, \alpha\right)}$ in (3.37) is given by

$$
L_{k}^{\left(\alpha^{\prime}, \alpha\right)}=\left(\boldsymbol{r}_{k}^{\alpha}\right)^{*} L_{S}(\boldsymbol{k}) \boldsymbol{r}_{k}^{\alpha^{\prime}},
$$

where the matrix symbol $L_{S}(\boldsymbol{k})$ associated with the slow operator $L_{S}$ is given, for $\boldsymbol{k} \neq \mathbf{0}$ and $\boldsymbol{k}=\mathbf{0}$, respectively, by

$$
L_{S}(\boldsymbol{k})=\frac{1}{F r}\left(\begin{array}{cccr}
0 & 0 & 0 & -\frac{k m}{|\boldsymbol{k}|^{2}} \\
0 & 0 & 0 & -\frac{l m}{|\boldsymbol{k}|^{2}} \\
0 & 0 & 0 & \frac{\left|\boldsymbol{k}_{H}\right|^{2}}{|\boldsymbol{k}|^{2}} \\
0 & 0 & -1 & 0
\end{array}\right), \quad L_{S}(\mathbf{0})=\frac{1}{F r}\left(\begin{array}{cccc}
0 & 0 & 0 & 0 \\
0 & 0 & 0 & 0 \\
0 & 0 & 0 & 1 \\
0 & 0 & -1 & 0
\end{array}\right) .
$$

Direct calculation of these coefficients with the eigenvectors given in (A 2) and (A 3) gives the explicit values of $L_{k}^{(0, \pm 1)}= \pm \mathrm{i} / \sqrt{2}$ and $L_{k}^{( \pm 1,0)}=\mp \mathrm{i} / \sqrt{2}$ when $\boldsymbol{k}=(k, l, 0)$, $L_{\mathbf{0}}^{(0,0)}=1$, and $L_{\mathbf{0}}^{(\tilde{0}, 0)}=-1$ when $\boldsymbol{k}=\mathbf{0}$, and zero otherwise.

Finally, the diffusion coefficient $D_{k}^{\left(\alpha^{\prime}, \alpha\right)}$ in (3.37) is given by

$$
D_{k}^{\left(\alpha^{\prime}, \alpha\right)}=\left(\boldsymbol{r}_{k}^{\alpha}\right)^{*} D(\boldsymbol{k}) \boldsymbol{r}_{k}^{\alpha^{\prime}}
$$

where $D(\boldsymbol{k})$ is the diagonal matrix

$$
D(\boldsymbol{k})=\operatorname{diag}\left(-\frac{1}{\operatorname{Re}}|\boldsymbol{k}|^{2}, \quad-\frac{1}{\operatorname{Re}}|\boldsymbol{k}|^{2}, \quad-\frac{1}{\operatorname{Re}}|\boldsymbol{k}|^{2}, \quad-\frac{1}{\operatorname{RePr}}|\boldsymbol{k}|^{2}\right),
$$

and direct calculation with the eigenvectors given in (A 2) and (A 3) shows that

$$
D_{k}^{(1,1)}=D_{k}^{(-1,-1)}=-\frac{1}{\operatorname{Re}}|\boldsymbol{k}|^{2}, \quad D_{k}^{(0,0)}=-\frac{1}{\operatorname{RePr}}|\boldsymbol{k}|^{2},
$$

and zero otherwise.

\section{REFERENCES}

Babin, A., Mahalov, A. \& Nicolaenko, B. 1996 Global splitting, integrability and regularity of 3D Euler and Navier-Stokes equations for uniformly rotating fluids. Eur. J. Mech. B/Fluids 15 (3), 291-300.

Babin, A., Mahalov, A., Nicolaenko, B. \& Zhou, Y. 1997 On the asymptotic regimes and the strongly stratified limit of rotating boussinesq equations. Theor. Comput. Fluid Dyn. 9 (3/4), $223-251$.

Babin, A., Mahalov, A. \& Nicolaenko 1998 On nonlinear baroclinic waves and adjustment of pancake dynamics. Theor. Comput. Fluid Dyn. 11 (3/4), 215-235. 
Babin, A., Mahalov, A. \& Nicolaenko, B. 2002 Fast singular oscillating limits of stably stratified three-dimensional Euler and Navier-Stokes equations and ageostrophic fronts. In Large-Scale Atmosphere-Ocean Dynamics (ed. J. Hunt, J. Norbury \& I. Roulstone) pp. 126-201. Cambridge University Press.

Charney, J. G. 1948 On the scale of atmospheric motions. Geofysiske Publikasjoner 17 (2), 3.

Chasnov, J. R. 1994 Similarity states of passive scalar transport in isotropic turbulence. Phys. Fluids 6, 1036-1051.

Chen, Q., Chen, S., Eyink, G. L. \& Holm, D. D. 2005 Resonant interactions in rotating homogeneous three-dimensional turbulence. J. Fluid Mech. 542, 139-164.

Davidson, P. A., Staplehurst, P. J. \& Dalziel, S. B. 2006 On the evolution of eddies in a rapidly rotating system. J. Fluid Mech. 557, 135-144.

Davies, P. A. 1972 Experiments on Taylor columns in rotating, stratified fluids. J. Fluid Mech. 54, 691-718.

EMBID, P. F. \& MAJDA, A. J. 1996 Averaging over fast gravity waves for geophysical flows with arbitrary potential vorticity. Commun. Part. Diff. Equ. 21 (3-4), 619-658.

EMBID, P. F. \& MAJDA, A. J. 1998 Low Froude number limiting dynamics for stably stratified flow with small or finite Rossby numbers. Geophys. Astrophys. Fluid Dyn. 87 (1-2), 1-50.

Emery, W. J., Lee, W. G. \& MagaArd, L. 1984 Geographic and seasonal distributions of BruntVaisala frequency and Rossby radii in the North Pacific and North Atlantic. J. Phys. Oceanogr. 14, 294-317.

Greenspan, H. P. 1990 The Theory of Rotating Fluids. Breukelen Press.

Heywood, K. J., Garabato, A. N. \& Stevens, D. P. 2002 High mixing rates in the abyssal Southern ocean. Nature 415, 1011-1014.

Jones, E. P., Rudels, B. \& Anderson, L. G. 1995 Deep waters of the Arctic Ocean: origins and circulation. Deep-Sea Res. 42, 737-760.

Kelvin, L. W. 1880 Vibrations of a columnar vortex. Phil. Mag. 10, 155-168.

Klainerman, S. \& MajdA, A. 1981 Singular limits of quasilinear hyperbolic systems with large parameters and the incompressible limit of compressible fluids. Commun. Pure Appl. Math. 34 (4), 481-524.

LAX, P. 2002 Functional Analysis. Wiley-Interscience.

LeBlond, P. H. \& MysaK, L. A. 1978 Waves in the Ocean. Elsevier Oceanography Series 20. Elsevier Scientific Publishing Company.

Majda, A. 1984 Compressible Fluid Flow and Systems of Conservation Laws in Several Space Variables. Springer-Verlag.

MajdA, A. J. \& Grote, M. J. 1997 Model dynamics and vertical collapse in decaying strongly stratified flows. Phys. Fluids 9 (10), 2932-2940.

MajdA, A. J. \& EmbID, P. 1998 Averaging over fast gravity waves for geophysical flows with unbalanced initial data. Theor. Comput. Fluid Dyn. 11 (3/4), 155-169.

Otheguy, P., Chomaz, J. \& Billant, P. 2006 Elliptic and zigzag instabilities on co-rotating vertical vortices in a stratified fluid. J. Fluid Mech. 553, 253-272.

Potylitsin, P. G. \& Peltier, W. R. 1998 Stratification effects on the stability of columnar vortices on the $f$-plane. J. Fluid Mech. 355, 45-79.

Potylitsin, P. G. \& Peltier, W. R. 2003 On the nonlinear evolution of columnar vortices in a rotating environment. Geophys. Astrophys. Fluid Dyn. 97, 365-391.

Riley, J. J. \& DEBruYnKops, S. M. 2003 Dynamics of turbulence strongly influenced by buoyancy. Phys. Fluids 15 (7), 2047-2059.

Riley, J. J. \& Lelong, M. P. 2000 Fluid motions in the presence of strong stable stratification. Annu. Rev. Fluid Mech. 32, 613-657.

SCHochet, S. 1987 Singular limits in bounded domains for quasilinear symmetric hyperbolic systems having a vorticity equation. J. Diff. Equ. 68 (3), 400-428.

Schochet, S. 1994 Fast singular limits of hyperbolic pde's. J. Dif. Equ. 114, 476-512.

Smith, L. M. \& WalefFe, F. 2002 Generation of slow large scales in forced rotating stratified turbulence. J. Fluid Mech. 451, 145-168.

Sreenivasan, B. \& Davidson, P. A. 2008 On the formation of cyclones and anticyclones in a rotating fluid. Phys. Fluids 20, 085104. 
Staplehurst, P. J., Davidson, P. A. \& Dalziel, S. B. 2008 Structure formation in homogeneous freely decaying rotating turbulence. J. Fluid Mech. 598, 81-105.

TaYlor, G. I. 1921 Experiments with rotating fluids. Proc. R. Soc. Lond. 100, 114-121.

Timmermans, M. L., Melling, H. \& Rainville, L. 2007 Dynamics in the deep Canada Basin, Arctic Ocean, inferred by thermistor-chain time series. J. Phys. Oceanogr. 37, 1066-1076.

Timmermans, M. L., Rainville, L., Thomas, L. \& Proshutinsky, A. 2010 Moored observations of bottom-intensified motions in the deep Canada Basin. J. Mar. Res. 68 (1), 625-641.

Woodgate, R., Aagaard, K., Muench, R., Gunn, J., Buörk, G., Rudels, B., Roach, A. T. \& Schauer, U. 2001 The Arctic Ocean boundary current along the Eurasian slope and the adjacent Lomonosov Ridge: Water mass properties, transports and transformations from moored instruments. Deep-Sea Res. 48, 1757-1892.

Van Haren, H. \& Millot, C. 2005 Gyroscopic waves in the Mediterranean Sea. Geophys. Res. Lett. $32(24), 1-4$. 